\title{
Superuniversality of Superdiffusion
}

\author{
Enej Ilievski, ${ }^{1}$ Jacopo De Nardis, ${ }^{2}$ Sarang Gopalakrishnan, ${ }^{3}$ Romain Vasseur, ${ }^{4}$ and Brayden Ware ${ }^{4}$ \\ ${ }^{1}$ Faculty for Mathematics and Physics, University of Ljubljana, Jadranska ulica 19, \\ 1000 Ljubljana, Slovenia \\ ${ }^{2}$ Department of Physics and Astronomy, University of Ghent, Krijgslaan 281, 9000 Gent, Belgium \\ ${ }^{3}$ Department of Physics and Astronomy, CUNY College of Staten Island, Staten Island, New York 10314; \\ Physics Program and Initiative for the Theoretical Sciences, The Graduate Center, \\ CUNY, New York, New York 10016, USA \\ ${ }^{4}$ Department of Physics, University of Massachusetts, Amherst, Massachusetts 01003, USA
}

(Received 24 October 2020; revised 4 May 2021; accepted 19 May 2021; published 28 July 2021)

\begin{abstract}
Anomalous finite-temperature transport has recently been observed in numerical studies of various integrable models in one dimension; these models share the feature of being invariant under a continuous non-Abelian global symmetry. This work offers a comprehensive group-theoretic account of this elusive phenomenon. For an integrable quantum model with local interactions, invariant under a global nonAbelian simple Lie group $G$, we find that finite-temperature transport of Noether charges associated with symmetry $G$ in thermal states that are invariant under $G$ is universally superdiffusive and characterized by the dynamical exponent $z=3 / 2$. This conclusion holds regardless of the Lie algebra symmetry, local degrees of freedom (on-site representations), Lorentz invariance, or particular realization of microscopic interactions: We accordingly dub it "superuniversal." The anomalous transport behavior is attributed to long-lived giant quasiparticles dressed by thermal fluctuations. We provide an algebraic viewpoint on the corresponding dressing transformation and elucidate formal connections to fusion identities amongst the quantum-group characters. We identify giant quasiparticles with nonlinear soliton modes of classical field theories that describe low-energy excitations above ferromagnetic vacua. Our analysis of these field theories also provides a complete classification of the low-energy (i.e., Goldstone-mode) spectra of quantum isotropic ferromagnetic chains.
\end{abstract}

DOI: 10.1103/PhysRevX.11.031023

Subject Areas: Statistical Physics

\section{INTRODUCTION}

A complete characterization and classification of dynamical properties of isolated interacting many-body systems remains one of the central unsettled problems in statistical mechanics. Especially in one-dimensional systems, a range of exotic dynamical phenomena have been demonstrated, both theoretically and experimentally. Two prominent examples are integrable and many-body localized quantum systems [1-3], which feature extensively many conserved quantities and therefore can persist in nonthermal "generalized Gibbs states" that are measurably different from the orthodox thermal ensemble [4-9]. Because these extensive conservation laws lead to nonstandard equilibrium states, and because hydrodynamics begins with an assumption of local thermal equilibrium, it follows that hydrodynamics is also modified for integrable

Published by the American Physical Society under the terms of the Creative Commons Attribution 4.0 International license. Further distribution of this work must maintain attribution to the author(s) and the published article's title, journal citation, and DOI. systems. Thus, instead of normal diffusion, nondisordered integrable systems typically exhibit ballistic transport with finite Drude weights [10,11], whereas in localized models, transport is entirely absent [2].

Integrable systems feature coherent quasiparticle excitations with an infinite lifetime [12,13] that propagate through the system in a ballistic manner while scattering elastically off one another. The same picture remains valid in thermal ensembles at finite temperature, where one can think of quasiparticles being "dressed" due to interactions with a macroscopic thermal environment [14]. Thermal fluctuations are responsible for screening, and thus conserved charges carried by quasiparticles can be appreciably different from the bare values. This effect is captured by the versatile framework of generalized hydrodynamics (GHD) $[15,16]$. Among other results, GHD has enabled the explicit characterization of ballistic transport [17-20] and analytic treatments of various other nonequilibrium protocols [21-25]. Remarkably, despite the ballistic motion of individual excitations, there are situations in which certain integrable models do not exhibit ballistic transport on macroscopic scales but instead display normal diffusion or even anomalous diffusion; this happens specifically for a 
distinguished subset of conserved quantities linked with internal degrees of freedom, whereas other conserved quantities (such as, e.g., energy) undergo ballistic transport [26].

In this work, we address the phenomenon of superdiffusive transport in integrable models. This unexpected phenomenon was first found in Ref. [29] in the Heisenberg spin- $1 / 2$ chain, an archetypal example of a quantum many-body interacting system. Nowadays, there is numerical evidence [30] that the dynamical exponent and asymptotic scaling profiles of dynamical structure factors belong to the Kardar-Parisi-Zhang (KPZ) universality class [31]. Specifically, the diagonal dynamical spin correlations $C^{j j}(x, t) \equiv\left\langle S^{j}(x, t) S^{j}(0,0)\right\rangle$ among the spin components $S^{j}$ (for all $j \in\{\mathrm{x}, \mathrm{y}, \mathrm{z}\}$ ), evaluated in thermal equilibrium at half filling, have been found to comply with the scaling form

$$
C^{j j}(x, t) \simeq \frac{C^{j j}}{\left(\lambda_{\mathrm{KPZ}} t\right)^{2 / 3}} f_{\mathrm{KPZ}}\left[\frac{x}{\left(\lambda_{\mathrm{KPZ}} t\right)^{2 / 3}}\right],
$$

characterized by the scaling function $f_{\mathrm{KPZ}}$ (tabulated in Ref. [32]) and the KPZ nonlinearity coupling parameter $\lambda$, and normalized by diagonal static charge susceptibilities $C^{j j}=\int d x C^{j j}(x, t)$. Kardar-Parisi-Zhang physics is a widespread phenomenon in stochastic growth processes and dynamical interfaces [33-36]. However, stochasticity is not of fundamental importance, and there are known cases of KPZ dynamics - most famously, the Fermi-PastaUlam-Tsingou model of coupled anharmonic oscillatorsalso in deterministic Hamiltonian dynamical systems. Even though the microscopic mechanism responsible for superdiffusion in the quantum Heisenberg chain (alongside other integrable models with non-Abelian symmetry as discussed here) is not yet understood in full detail, it nevertheless seems to fall outside of the conventional framework of nonlinear fluctuating hydrodynamics (NLFHD) that describes the underlying mode-coupling mechanism in nonintegrable models with a finite number of local conservation laws. The fact that spin (or charge) superdiffusion of the KPZ type has also been numerically observed in a number of other quantum chains-such as the higher-spin $\mathrm{SU}(2)$ integrable chains and the $\mathrm{SO}(5)$ symmetric spin ladder [37], the Fermi-Hubbard model [38], and even in the classical Landau-Lifshitz equation [39-41] (and its higher-rank analogues that exhibit symmetry of non-Abelian unitary groups [42])—gives a strong indication that there is a general principle behind superdiffusion in integrable systems with non-Abelian symmetries that is yet to be uncovered.

GHD has already provided a number of invaluable theoretical insights into this question. Specifically, for the case of the quantum Heisenberg spin chains, both the dynamical exponent $z=3 / 2$ [43] and the KPZ coupling constant $\lambda_{\mathrm{KPZ}}$ [44] (though not the scaling function) have been inferred with the aid of a heuristic extension of the GHD framework, taking full advantage of the exact knowledge of the Bethe ansatz quasiparticles. Recently, a phenomenological explanation for the observed KPZ phenomenon has been given in Ref. [45], which invokes the notion of hydrodynamic "soft gauge modes" coupled to an effective noise stemming from thermal fluctuations of other hydrodynamic modes. The current understanding is that such soft models are a manifestation of the so-called giant quasiparticles in the long-wavelength regime, whose emergent dynamics is governed by a classical action [44]. This idea appears to suggest that the observed superdiffusive spin dynamics in classical rotationally symmetric [e.g., $\mathrm{SO}(3)$-invariant] spin chains has the same microscopic origin as that of quantum spin chains. In spite of this theoretical progress, however, a fundamental question nonetheless remains unsettled: In integrable Hamiltonian dynamical systems, what are the necessary and sufficient conditions for the superdiffusive charge dynamics characterized by anomalous dynamical exponent $z=3 / 2$ ? We dedicate this paper to answering this question on general grounds, in both integrable lattice models and integrable quantum field theories.

Before delving into mostly technical aspects, we offer a broader perspective on the problem at hand. By invoking the universal GHD formulas for the spin diffusion constants, it is evident from the outset that divergent charge diffusion constants are only possible in systems with infinitely many types of quasiparticles in the spectrum. This necessary condition is quite generally fulfilled in integrable lattice models, including, in particular, the class of models with non-Abelian symmetries, which is our primary focus. Given that such models $d o$ have infinitely many quasiparticle types (owing to formation of bound states), however, there are two scenarios that seem particularly plausible:

(I) All integrable Hamiltonians that are symmetric under a non-Abelian Lie group $G$ display universal superdiffusive dynamics of the Noether charges in $G$-invariant (i.e., unpolarized) Gibbs states.

(II) Integrable models allow for a wider range of dynamical exponents, depending, e.g., on the rank or type of Lie algebras and their representations assigned to local degrees of freedom.

There are several recent studies, e.g., Refs. [37,38,46], which offer evidence in favor of scenario (I). Another strong piece of evidence comes from a recent study [42] of transport properties in classical integrable matrix models in discrete space-time, representing discretizations of higherrank analogues of $\mathrm{SU}(n)$-symmetric Landau-Lifshitz field theories on complex projective spaces and Grassmannian manifolds. Besides providing convincing numerical evidence for the KPZ scaling profiles independently of rank $r=n-1$, Ref. [42] speculates that the same phenomenon will persist in all classical (nonrelativistic) $G$-invariant integrable field theories whose target manifolds belong 
to Hermitian symmetric spaces. Indeed, the class of models considered in Ref. [42] governs low-energy spectra of integrable $\mathrm{SU}(n)$-invariant Lai-Sutherland ferromagnetic spin chains $[47,48]$ that are included as part of our survey. On this basis, it is reasonable to anticipate that coherent semiclassical modes analogous to the aforementioned giant quasiparticles emerge as a general feature of integrable quantum chains. Nevertheless, their microscopic description is not known at this moment.

However, option (II) cannot be a priori rejected either. We note that the phenomenological framework of nonlinear fluctuating hydrodynamics $[49,50]$ allows for, in principle at least, an infinite tower of anomalous dynamical exponents [51]. Now, NLFHD does not directly apply to integrable systems, and there are good reasons to doubt that it can capture superdiffusion in the integrable case [45]: Notably, in NLFHD, superdiffusion arises as a correction to ballistic transport, rather than as the leading dynamical behavior. Regardless, the existing numerical evidence on dynamical exponents in integrable systems is also ambiguous: For instance, a study of the integrable SU(4) spin chain found a distinct exponent $z \approx 5 / 3$ [52], suggesting the possibility that different non-Abelian symmetries may, after all, realize distinct universality classes of anomalous transport. A key result of our work will be to show, instead, that $z=3 / 2$ is superuniversal and that there is no room for a different exponent such as $z=5 / 3$. We also provide improved numerics showing that transport in SU(4) spin chains (and in quantum chains with other non-Abelian Lie groups) is in fact consistent with $z=3 / 2$. In addition to its fundamental interest, this question is experimentally relevant because interacting quantum lattice systems possessing $\mathrm{SU}(N)$ symmetry can be implemented using ultracold alkaline-earth atoms $[53,54]$. In the context of solid-state physics, several strongly coupled ladder compounds also realize (approximately) the $\mathrm{SU}(4)$-symmetric ladder in the presence of fields [55-57].

\section{A. Summary}

In this work, we outline a systematic theoretical analysis of anomalous charge transport, specializing to the class of integrable quantum "spin chains" that exhibit global symmetries of non-Abelian simple Lie groups. Our analysis partially relies on a universal algebraic description linked to representation theory of quantum groups (and the associated fusion relations). For the reader's convenience, our focus will be mainly to collect and discuss all the relevant statements while relating the largely technical material to Ref. [58]. For clarity, we first summarize our main findings.

Our central result is that an anomalous algebraic dynamical exponent $z=3 / 2$ associated with transport of the Noether charges is a common feature of integrable Hamiltonian systems invariant under a general non-Abelian Lie group $G$, including both quantum spin chains with local interactions and quantum field theories. The key extra requirement is that the equilibrium state does not break the global symmetry by the presence of finite chemical potentials. This statement holds independently of the type of simple Lie algebra or the unitary representations associated with local Hilbert spaces (in quantum chains) or local degrees of freedom (in integrable QFTs): The only requirement is that the charge $Q$ whose correlation functions we study must transform nontrivially under $G$ (unlike, e.g., the energy). We thereby establish superuniversality of superdiffusive charge transport.

Our analysis incorporates the following complementary approaches:

(i) We carry out a scaling analysis of the universal nested Bethe ansatz dressing equations, concluding that the spectrum of "giant quasiparticles" exhibits the same type of asymptotic scaling relations irrespectively of the non-Abelian symmetry algebra. This conclusion implies that the kinetic theory argument outlined in Ref. [43] carries through, in general. To solidify this conclusion, we derive an explicit closed-form solution of the dressing equations for the case of higher-rank unitary groups $\mathrm{SU}(N)$ [including the general dependence on the U(1) chemical potentials coupling to the Cartan charges].

(ii) Second, we verify our predictions through tensornetwork-based numerical simulations by computing dynamical charge correlation functions for a number of representative cases, including unitary, orthogonal, and symplectic Lie groups (shown in Figs. 1 and 2 ); evidently, all the cases we have studied yield the exponent $z=3 / 2$.

(iii) Lastly, we elucidate the physical nature of the giant quasiparticles. The latter govern the semiclassical long-wavelength dynamics of the charge density in highly excited eigenstates. From the spin chain viewpoint, they correspond to macroscopically large coherent states made out of interacting, quadratically dispersing (i.e., "magnonlike"), Goldstone modes above a ferromagnetic vacuum. When viewed as classical objects, they are none other than localized nonlinear modes called solitons. Although it may seem counterintuitive (at first glance at least) that long-wavelength modes matter to high-temperature physics, we notice that it is precisely due to integrability that these large bound-state excitations remain well-defined quasiparticles even away from the vacuum and also in thermal states in the presence of other modes; however, their (bare) properties get dressed by thermal fluctuations. To corroborate this, we establish a one-to-one correspondence between the Bethe ansatz quasiparticles and the spectrum of Goldstone modes. This correspondence is subtle: There are many more distinct Goldstone modes for a given symmetry-breaking pattern than there are magnon species (flavors) in the Bethe ansatz spectrum. Therefore, counting the Goldstone modes 

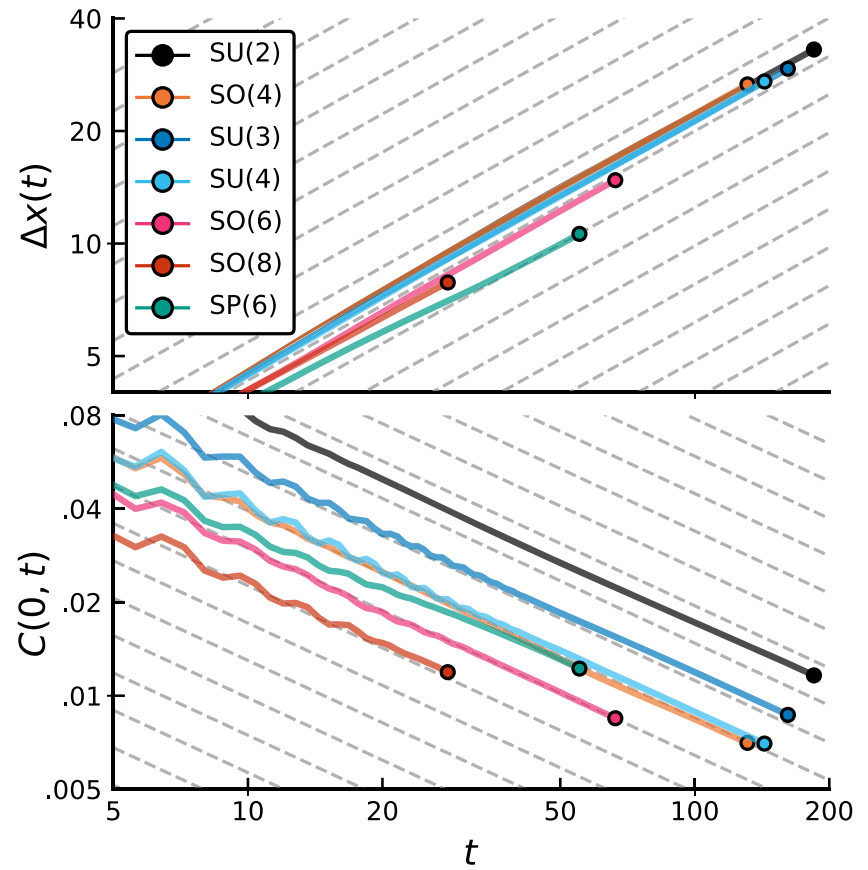

FIG. 1. Dynamical charge correlation functions computed with TEBD, showing asymptotic scaling with dynamical exponent $z=$ $3 / 2$ for each of the integrable chains, measured by (top) the width $\Delta x(t)$ of the expanding charge profiles and (bottom) the return probability $C(0, t)$. The dashed lines in the background show $\Delta x \propto t^{2 / 3}$ and $C(0, t) \propto t^{-2 / 3}$.

correctly requires the notion of composite quasiparticles called "stacks." To our knowledge, these multiflavored stacks have not been explicitly classified thus far.

While we mostly focus on integrable quantum chains with ferromagnetic exchange, we also briefly discuss, in the Appendix C, how KPZ superuniversality emerges even in integrable quantum field theories possessing internal isotropic degrees of freedom (which take values in compact simple Lie groups $G$ or coset spaces thereof), both for Lorentz-invariant theories and for nonrelativistic interacting fermions. Such integrable QFTs are characterized by nondiagonal scattering, signifying that their elementary quasiparticle excitations can exchange isotropic degrees of freedom upon elastic collisions. This process results in dynamically produced massless pseudoparticles that are responsible for charge transport at finite temperature. These so-called "auxiliary quasiparticles" are interacting magnon waves and bound states thereof, which formally resemble magnons of the quantum ferromagnetic spin chains [59]. Divergent charge diffusion constants are thus, once again, attributed to the presence of interacting giant magnons.

Outline. The paper is structured as follows. In Sec. II, we succinctly review the formalism of generalized hydrodynamics, provide closed formulas for the charge diffusion constants, and proceed by summarizing the general physical picture and scaling arguments that lead to

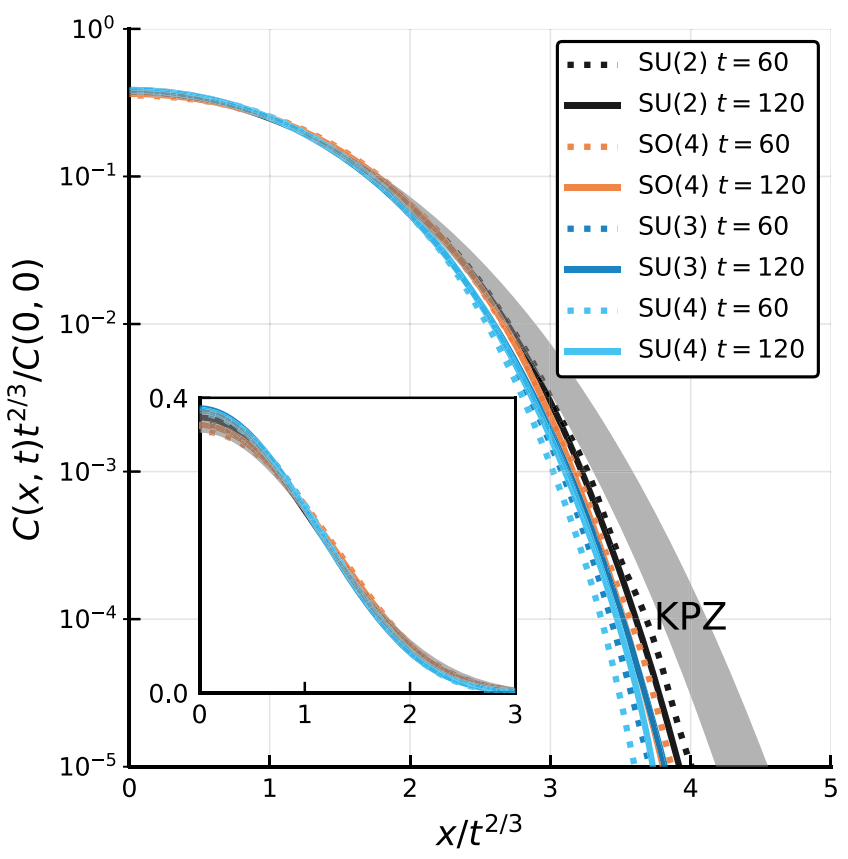

FIG. 2. Asymptotic charge correlator profiles rescaled using the dynamical exponent $z=3 / 2$ collapse. The highlighted region shows the KPZ scaling function for a range of widths fit to these profiles - the fit fails, as the tails of the scaling function appear to fall off faster than in KPZ scaling. The inset shows the same plot with a linear axis.

superuniversality. Technical derivations are provided in the Supplemental Material [58], which contains detailed information about the nested Bethe ansatz diagonalization technique and the algebraic structure of the quasiparticle spectra for a family of quantum spin chains, including Refs. [59-109]. In Sec. III, we discuss our numerical results on transport, while Sec. IV is devoted to the semiclassical limit of integrable models and related concepts. In Sec. V, we summarize our results and propose areas for future exploration.

\section{SUPERDIFFUSION FROM GIANT QUASIPARTICLES}

We aim to characterize charge dynamics in Hamiltonian systems invariant under a non-Abelian continuous symmetry group $G$. We begin our presentation by first outlining the general setting and introducing the key quantities of the linear transport theory. We provide compact spectral representations for the charge diffusion constant in terms of quasiparticle spectra, largely following previous works on the subject $[43,109-112]$.

We specialize to simple Lie groups $G$ of rank $r$, generated by Lie algebra g. Owing to the Noether theorem, the system possesses local conserved (Noether) charges,

$$
Q^{(\sigma)}=\int d x q^{(\sigma)}(x),
$$


with local densities $q^{(\sigma)}(x)$, one for each Hermitian generator $\mathrm{X}^{\sigma}$ of Lie algebra $\mathrm{g}$. For simplicity of notation, we do not make explicit distinctions between lattice and continuum models; i.e., in lattice models, spatial integration in Eq. (2) should be understood as a discrete summation.

Our first objective is to obtain closed-form expressions for transport coefficients in equilibrium. We specialize to thermal states characterized by grand-canonical Gibbs ensembles (at inverse temperature $\beta$ ), corresponding to density matrices of the form

$$
\varrho_{\beta, \mathbf{h}}=\frac{1}{\mathcal{Z}_{\beta, \boldsymbol{h}}} \exp \left[-\beta H+\sum_{i=1}^{r} h_{i} Q^{(i)}\right] .
$$

Here, parameters $\mathbf{h} \equiv\left\{h_{i} \in \mathbb{R}\right\}$ are the $\mathrm{U}(1)$ chemical potentials that have been assigned to a maximal set of commuting (Cartan) charges $Q^{(i)}$ with $i \in \mathcal{I}_{r} \equiv\{1,2, \ldots, r\}$, while normalization $\mathcal{Z}_{\beta, \boldsymbol{h}} \equiv \operatorname{Tr} \varrho_{\beta, \mathbf{h}}$ represents the partition sum. Our results generalize to an arbitrary generalized Gibbs ensemble, including other conserved charges preserving the symmetry.

The presence of finite chemical potentials $\mathbf{h}$ allows us to study transport at generic values of the charge densities. When all $h_{i} \neq 0$, the ensembles (3) explicitly violate the global symmetry of $G$, and one is left with a residual symmetry of the maximal Abelian subgroup $T \equiv \mathrm{U}(1)^{r} \subset$ $G$ (called the maximal torus). The complete set of $\operatorname{dim}(\mathfrak{g})$ Noether charges can accordingly be separated into two sectors: the "longitudinal" charges $Q^{(i)}$ assigned to the "unbroken" (Cartan) generators and the non-Abelian set of "transversal" charges $Q^{(\sigma)}$ for $\sigma \notin \mathcal{I}_{r}$, satisfying $\left[Q^{(i)}, Q^{(\sigma \notin \mathcal{I})}\right] \neq 0$, associated with the "broken" generators. In this work, we are exclusively interested in emergent anomalous charge transport that arises in the limit $h_{i} \rightarrow 0$ where the distinction between longitudinal and transversal correlators disappears (since $G$ invariance of $\varrho_{\beta, \mathbf{h}}$ gets restored); it will thus be sufficient to focus exclusively on the longitudinal sector (see, e.g., Ref. [113] for remarks about the nature of transversal correlators).

\section{A. Generalized hydrodynamics}

We now formulate the transport theory for integrable systems, known as generalized hydrodynamics $[15,16]$ (cf. Ref. [114] for an overview). As our starting point, we begin by an infinite tower of local (including quasilocal $[9,104])$ conservation laws

$$
\partial_{t} q_{k}(x, t)+\partial_{x} j_{k}(x, t)=0 .
$$

For the time being, $k \in \mathbb{N}$ is just a formal discrete mode index. The complete set of (quasi)local charges can be constructed explicitly with the aid of the algebraic Bethe ansatz techniques as in Ref. [104]. The associated currents can be constructed in a similar spirit; see, e.g.,
Refs. $[115,116]$. We note that, for practical considerations, these steps can be entirely circumvented as long as one operates at the level of thermal averages.

The GHD formalism provides an explicit prescription to describe large-scale spatiotemporal variations of the densities of conserved charges, as provided by the local continuity equations (4). The key ingredient is to express expectation values of the current densities as functionals of the charge averages. While there exist various thermodynamic state functions to choose from, a particularly convenient one is to use quasiparticle rapidity densities $\rho_{A}(\theta)$; we have introduced the "type" label $A$, running over the entire model-specific (thermodynamic) quasiparticle content, and the rapidity variable $\theta$, which parametrizes their bare momenta, that is, $p_{A}=p_{A}(\theta)$. Importantly, an infinite collection of functions $\left\{\rho_{A}(\theta)\right\}$ uniquely specifies a macrostate, physically representing unbiased microcanonical ensembles of locally indistinguishable thermodynamic eigenstates. Furthermore, by exploiting a one-to-one correspondence between the expectation values of the (quasi)local conservation laws and quasiparticle content in an equilibrium macrostate [100,101], it proves useful to recast Eq. (4) as a continuity equation for the quasiparticle densities

$$
\partial_{t} \rho_{A}(\theta ; x, t)+\partial_{x} j_{A}(\theta ; x, t)=0 .
$$

The quasiparticle current densities take a simple factorized form at the Euler scale $[15,16]$,

$$
j_{A}(\theta)=v_{A}^{\mathrm{eff}}(\theta) \rho_{A}(\theta) .
$$

Here, $v_{A}^{\text {eff }}$ are state-dependent effective group velocities determined from the dressed quasiparticle dispersions

$$
v_{A}^{\mathrm{eff}}(\theta)=\frac{\partial_{\theta} \varepsilon_{A}(\theta)}{\partial_{\theta} p_{A}(\theta)}
$$

For a simple Lie group $G$ of rank $r$, elementary quasiparticle excitations (defined with respect to a reference ferromagnetic order parameter) come in exactly $r$ different "flavors." In addition, quasiparticles participate in the formation of bound states; a quasiparticle that carries $s$ quanta of flavor $a$ is accordingly assigned a pair of quantum numbers $A=(a, s)$. Therefore, the presence of infinitely many bound states, where $s$ takes values in an infinite countable set (typically ranging from 1 to $\infty$ ), is a common feature for all the models we consider subsequently.

Expanding above a reference equilibrium state with densities $\rho_{A}(\theta)$, that is, $\rho_{A}(\theta ; x, t)=\rho_{A}(\theta)+\delta \rho_{A}(\theta ; x, t)$, the hydrodynamic evolution of density (or charge) fluctuations $\delta \rho_{A}(\theta ; x, t)$ on the Euler scale is encoded in the linear propagator (flux Jacobian) $\mathbf{A}=\partial \mathbf{j} / \partial \boldsymbol{\rho}$, reading

$$
\partial_{t} \delta \boldsymbol{\rho}(x, t)+\partial_{x}(\mathbf{A} \delta \boldsymbol{\rho})=0,
$$


where (for compactness of presentation) we have employed the tensor notation by flattening the quasiparticle and rapidity labels. Equation (8) can be diagonalized by performing a basis transformation

$$
\delta \phi=\boldsymbol{\Omega}[\mathbf{n}] \delta \boldsymbol{\rho},
$$

depending (nonlinearly) on Fermi functions $\mathbf{n}$ of the underlying equilibrium state, where $\delta \phi$ are the normal modes of GHD (defined uniquely modulo normalization of static covariances). In the basis of normal modes, the propagator $\mathbf{A}$ acts diagonally, with eigenvalues given by the effective velocities (7), that is,

$$
\mathbf{\Omega A} \boldsymbol{\Omega}^{-1}=\mathbf{v}^{\mathrm{eff}} .
$$

The physical interpretation of $\boldsymbol{\Omega}[\mathbf{n}]$ becomes transparent in the formalism of the thermodynamic Bethe ansatz, where it plays the role of a dressing operator for conserved quantities,

$$
\mathbf{q}^{\mathrm{dr}}=\mathbf{\Omega}[\mathbf{n}] \mathbf{q} .
$$

\section{B. Diffusion constants}

We now introduce the linear transport coefficients. Here, we define them based on the asymptotic behavior of dynamical structure factors (or dynamical charge susceptibilities). For our purpose, it will be sufficient to only consider the Cartan charges and introduce the associated $r$-dimensional matrix of dynamical susceptibilities

$$
C^{i j}(x, t) \equiv\left\langle q^{(i)}(x, t) q^{(j)}(0,0)\right\rangle,
$$

where the brackets $\langle\bullet\rangle$ designate the connected correlator evaluated in a grand-canonical Gibbs ensemble given by Eq. (3). Static susceptibilities are accordingly given by the time-invariant sum rule $C^{i j}=\int d x C^{i j}(x, t)$.

For generic values of the Cartan chemical potentials $h_{i}$, the variance of the structure factor in integrable models experiences ballistic spreading,

$$
\int d x x^{2} C^{i j}(x, t) \simeq \mathcal{D}^{i j} t^{2 / z},
$$

signaled by the ballistic dynamical exponent $z=1$ and charge Drude weights $\mathcal{D}^{i j}[10,117,118]$. The charge Drude weights admit the following mode resolution $[18,19]$ :

$$
\mathcal{D}^{i j}=\sum_{A} \int d \theta \rho_{A}(\theta) \bar{n}_{A}(\theta)\left(v_{A}^{\mathrm{eff}}(\theta)\right)^{2} q_{A}^{(i) \mathrm{dr}} q_{A}^{(j) \mathrm{dr}} .
$$

Here, $\bar{n}_{A}(\theta) \equiv 1-n_{A}(\theta)$ denote thermal Fermi occupation functions associated with vacancies (holes). All the thermodynamic quantities in the integrand of Eq. (14) depend on temperature and chemical potentials.
Drude weights do not provide complete information about the late-time relaxation of $C^{i j}(x, t)$. To deduce the asymptotic behavior of $C^{i j}(x, t)$ on a sub-ballistic scale, we adopt the kinetic theory framework of Refs. [43,111]. One normally envisions a thermodynamic system divided up into large fluid cells of size $\ell$, with each cell being approximately in local thermal equilibrium. In a hydrodynamic description, both the dressed charges $q_{A}^{(i) \mathrm{dr}}$ and local chemical potentials $h_{i}$ get promoted to dynamical quantities, which, in a macroscopic fluid cell of length $\ell$, exhibit thermal fluctuations of the order $\mathcal{O}\left(\ell^{-1 / 2}\right)$, which will, in turn, lead to diffusion.

In generic local equilibrium states, diffusion is a subleading correction to the ballistic transport characterized by the Drude weight. However, in unpolarized thermal ensembles in systems with non-Abelian symmetries, the charge Drude weight vanishes and the leading transport behavior is sub-ballistic. Charge Drude weights are proportional to dressed charges $q_{A}^{(i) \mathrm{dr}}$ carried by quasiparticles, cf. Eq. (14). The latter are quite different from their bare (quantized) values $q_{A}^{(i)}$ and depend nontrivially on chemical potentials of the background equilibrium state, including the U(1) chemical potentials $h_{i}$. In unpolarized thermal states that exhibit full invariance under $G$, the dressed quasiparticle charges vanish simply by symmetry under $G$ (see, e.g., Refs. [17,19,45]): One can see this as the screening of the quasiparticle charge by the thermal environment. Therefore, the charge Drude weight (14) vanishes.

The leading response therefore occurs at the diffusive scale, where one treats the chemical potentials as dynamically fluctuating quantities, with fluctuations that are suppressed by the hydrodynamic scale, $\mathcal{O}\left(\ell^{-1 / 2}\right)$. For sufficiently small $h_{i}$, the quasiparticles carry dressed charges linearly proportional to $h_{i}$; i.e., they behave paramagnetically. Fluctuations of chemical potentials induce fluctuations of thermally dressed charges in accordance with

$$
q_{A}^{(i) \mathrm{dr}} q_{A}^{(j) \mathrm{dr}}=\left.\frac{1}{2} \sum_{k, l} \frac{\partial^{2}\left(q_{A}^{(i) \mathrm{dr}} q_{A}^{(j) \mathrm{dr}}\right)}{\partial h_{k} \partial h_{l}}\right|_{\mathbf{h} \rightarrow \mathbf{0}} h_{k} h_{l}+\ldots
$$

Notice that the chemical potentials can be simply related to densities of the Cartan charges via

$$
h_{k} h_{l}=\sum_{i, j}\left(C^{-1}\right)^{k i}\left(C^{-1}\right)^{l j} q^{(i)} q^{(j)} .
$$

Here, $\quad C^{i j}=\partial q^{(i)} / \partial h_{j}=\left(\partial^{2} / \partial h_{i} \partial h_{j}\right) \log \mathcal{Z}$ are static charge susceptibilities, and it will be helpful below to express them in terms of a mode expansion [18,19] analogous to Eq. (14):

$$
C^{i j}=\sum_{A} \int d \theta \rho_{A}(\theta) \bar{n}_{A}(\theta) q_{A}^{(i) \mathrm{dr}} q_{A}^{(j) \mathrm{dr}} .
$$


The latter also determine the magnitude of charge fluctuations, $\left\langle q^{(i)} q^{(j)}\right\rangle=C^{i j} / \ell$. By combining these two results, thermal fluctuations of dressed charges (or dressed susceptibilities) carried by individual quasiparticle modes can be expressed in the form

$$
\left\langle q_{A}^{(i) \mathrm{dr}} q_{A}^{(j) \mathrm{dr}}\right\rangle_{\mathbf{h} \rightarrow \mathbf{0}}=\frac{\Upsilon_{A}^{i j}}{\ell},
$$

where

$$
\Upsilon_{A}^{i j}=\left.\frac{1}{2} \sum_{k, l}\left(C^{-1}\right)^{k l} \frac{\partial^{2}\left(q_{A}^{(i) \mathrm{dr}} q_{A}^{(j) \mathrm{dr}}\right)}{\partial h_{k} \partial h_{l}}\right|_{\mathbf{h} \rightarrow \mathbf{0}}=C^{i j} \Upsilon_{A} .
$$

The quantities $\Upsilon_{A}^{i j}$ can be physically interpreted as effective paramagnetic moments, assuming nontrivial dependence on both quasiparticle quantum numbers $A=(a, s)$.

In diffusive dynamics, the variance of the dynamical structure factor $C^{i j}$ experiences linear growth at late times,

$$
\int d x C^{i j}(x, t) \simeq 2 \sigma^{i j} t
$$

characterized by the charge conductivity matrix

$$
\sigma=D C,
$$

with static susceptibility matrix $C$ and charge diffusion matrix $D$ [119]. A full expression for the conductivity (Onsager) matrix $\sigma$ has been derived in Refs. [110,112] using the form-factors approach, and its diagonal part in Ref. [111] using a kinetic theory formulation. Here, we provide a compact expression for $D$ in models of higherrank symmetry (restricted to the Cartan sector), specializing to the limit of vanishing chemical potentials, $\mathbf{h} \rightarrow \mathbf{0}$. To this end, we substitute the fluctuation relation (18) into Eq. (13) with Eq. (14) on a characteristic scale $\ell$ set by quasiparticles' effective velocities, $\ell=\left|v_{A}^{\text {eff }}(\theta)\right| t$ [43], yielding the following spectral resolution of the conductivity matrix:

$$
\sigma^{i j}(\mathbf{h}=\mathbf{0})=\frac{1}{2} \sum_{A} \int d \theta \rho_{A}(\theta) \bar{n}_{A}(\theta)\left|v_{A}^{\mathrm{eff}}(\theta)\right| \Upsilon_{A}^{i j} .
$$

The diffusion matrix is thus proportional to the identity, corresponding to a single value of charge diffusion constant

$$
D=\frac{1}{2} \sum_{A} \int d \theta \rho_{A}(\theta) \bar{n}_{A}(\theta)\left|v_{A}^{\mathrm{eff}}(\theta)\right| \Upsilon_{A} \cdot
$$

The quantities $\Upsilon_{A}^{i j}$ can be easily computed explicitly at infinite temperature in various integrable spin chains (see Appendixes). For instance, in the $A_{n} \equiv \mathrm{SU}(n)$ ferromagnetic integrable chains, we find $\Upsilon_{s}^{A_{1}}=\frac{4}{9}(s+1)^{4}$, $\Upsilon_{a, s}^{\mathrm{A}_{2}}=\frac{1}{16}(1+s)^{2}(2+s)^{2}$, while for $n \geq 4$, it depends on the flavor label $a \in\{1,2, \ldots, n-1\}$. Its precise form will not matter for our purposes, but note the large-s scaling $\Upsilon_{A} \sim s^{4}$.

By adopting quasiparticle densities as variational objects (see Appendix A for the derivation) and comparing the result to Eq. (22), we deduce the following relation between the dressed charge fluctuations and the dressed differential scattering phases $K_{A A^{\prime}}^{\mathrm{dr}}$,

$\left\langle q_{a, s}^{(i) \mathrm{dr}} q_{a, s}^{(i) \mathrm{dr}}\right\rangle_{\mathbf{h} \rightarrow \mathbf{0}}=\frac{C^{i i}}{\ell}\left[\sum_{a^{\prime}} q_{a^{\prime}}^{(i)} \lim _{s^{\prime} \rightarrow \infty} s^{\prime} K_{a s, a^{\prime} s^{\prime}}^{\mathrm{dr}}\right]^{2}$.

Thus, we get the following nontrivial identity:

$$
\Upsilon_{a, s}=\left[\sum_{a^{\prime}} q_{a^{\prime}}^{(i)} \lim _{s^{\prime} \rightarrow \infty} s^{\prime} K_{a s, a^{\prime} s^{\prime}}^{\mathrm{dr}}\right]^{2}
$$

We remark that this expression can be thought of as a generalization of the so-called "magic formula" of Ref. [109], which was originally introduced as a way to relate the different expressions of the diffusion constant of the $X X Z$ spin chain with interaction anisotropy $\Delta>1$ obtained from form factors [112] and from a kinetic theory argument [43] similar to the one we used above.

\section{Emergence of superdiffusion}

From the considerations above, one might expect to find normal diffusion with $z=2$, with the diffusion constant given by Eq. (22). Explicit computation shows, however, that this result diverges as $h_{i} \rightarrow 0[43,46]$. This divergence has been catalogued for various specific cases; here, we explain why it holds, in general. To this end, we define the regularized diffusion constant by truncating the spectral sum (22) at some string index $s_{*}$,

$$
D_{*}=\sum_{a=1}^{r} \sum_{s=1}^{s_{*}} D_{a, s}
$$

where $D_{a, s} \equiv D_{A}$ corresponds to each summand in Eq. (22). As a finite sum, Eq. (26) is manifestly finite. If $D_{*}$ converges as $s_{*} \rightarrow \infty$, then one has normal diffusion.

First, we observe that the convergence properties of $D_{*}$ are tightly linked to those of the static susceptibility. As $\mathbf{h} \rightarrow \mathbf{0}$, the static charge susceptibilities $C^{i j}$, given by Eq. (17), must approach a constant value. At finite $\mathbf{h}$, the occupation factor for a quasiparticle of type $(a, s)$ (a bound state of $s$ magnons of flavor $a$ ) is suppressed with a factor of $\exp \left(-s h_{a}\right)$, and thus quasiparticles with $s h_{a} \gg 1$ do not contribute to the susceptibility at chemical potential $h_{a}$. The label " $a$ " essentially plays no role in the following argument, so we drop it. Uniformly writing $h_{a} \rightarrow h$, truncating the sum (17) at $s \sim 1 / h$, and using the paramagnetic behavior of the dressed charges, we have $C^{i j} \sim h^{2} \sum_{a=1}^{r} \sum_{s<1 / h} C_{a, s}^{i j}$. Requiring that the $C^{i j}$ have a finite yet nonvanishing limit as $h \rightarrow 0$, we see that the sum 
over $s$ has to scale as about $s^{2}$, so the summand $C_{a, s}^{i j}$ must grow linearly in $s$. Crucially, however, the summands in Eqs. (17) and (22) are the same, except for a factor of the velocity. Suppose the characteristic velocity of a quasiparticle with labels $s$ scales as $\left|v_{s}^{\text {eff }}\right| \sim s^{-\nu}$. Then, the diffusion constant must scale as

$$
D_{*} \sim \sum_{s \leq s^{*}} s^{1-\nu} \sim s^{2-\nu}, \quad \nu<2 .
$$

If $\nu<2$, the diffusion constant diverges, yielding superdiffusive transport, whereas if $\nu>2$, it converges and one has diffusive transport. (In the marginal case $\nu=2$, one has a logarithmically divergent diffusion constant [120].)

Next, we relate this divergence of $D_{*}$ to the time dependence of transport. Recall that the dynamical critical exponent $z$ is defined by the relation $x^{2} \sim t^{2 / z}$. Following standard notation, we define a time-dependent diffusion constant via the relation $x^{2} \sim D(t) t$, and (by equating the two relations above) we observe that

$$
D(t) \sim t^{(2 / z)-1} .
$$

We now apply the kinetic argument [43] to relate the exponents $\nu$ and $z$. At time $t$, we can divide quasiparticles into "light" quasiparticles for which $v_{s} t \sim t / s^{\nu}>x(t) \sim$ $t^{1 / z}$ and "heavy" quasiparticles for which $v_{s} t<t^{1 / z}$. The crossover between light and heavy quasiparticles takes place when $v_{s} t \sim t / s^{\nu} \sim t^{1 / z}$. Thus, we have that $s_{*} \sim t^{(1-1 / z) / \nu}$. Plugging this result into Eq. (27), we arrive at the conclusion

$$
D(t) \sim D\left(s_{*}(t)\right) \sim\left[t^{(1-1 / z) / \nu}\right]^{2-\nu} .
$$

Equating the exponents in Eqs. (28) and (29), we find the following relation between the exponents $z$ and $\nu$ :

$$
z=\left(1+\frac{\nu}{2}\right), \quad \nu<2
$$

From the analytic structure of the Bethe ansatz solutions, one can infer that the exponent $\nu \geq 0$ is integer valued. This fact rules out all but three possible values for $z$. The three remaining possibilities are ballistic transport $(\nu=0)$, which is ruled out by the vanishing Drude weight discussed above; KPZ scaling $(\nu=1)$, which we will argue is generic; and diffusion with possible logarithmic corrections $(\nu \geq 2)$, which occurs in certain fine-tuned models [120] where the single-magnon dispersion is fine-tuned to scale as $\omega \sim$ $k^{3}$ (or slower) at long wavelengths $k \rightarrow 0$. The only superdiffusive exponent that is allowed by the structure of these integrable models is $z=3 / 2$.

In what follows, we will establish $z=3 / 2$ within a universal algebraic description of the thermodynamic dressing equations and link them to the representation theory of the underlying symmetry structures-quantum groups called Yangians, in particular. We find, remarkably, that the Fermi functions assume universal algebraic scaling at large $s$,

$$
n_{a, s} \sim \frac{1}{s^{2}},
$$

which is a direct corollary of fusion identities amongst the quantum characters associated to the Yangian symmetry [67-69,121,122]. Similarly, we find that the total state densities and the dressed velocities of giant magnons (when multiplied by a regular function and integrated over the rapidity domain [58]) decay as

$$
\rho_{a, s}^{\mathrm{tot}}=\frac{\rho_{a, s}}{n_{a, s}} \sim \frac{1}{s} \text { and } v_{a, s}^{\mathrm{eff}} \sim \frac{1}{s} \text {, }
$$

respectively, for all flavors $a=1, \ldots, r$. Most remarkably, these large- $s$ scaling properties hold irrespectively of the local on-site degrees of freedom (i.e., finite-dimensional, irreducible, unitary representations of $\mathfrak{g}$ ); they can be understood as kinematic constraints stemming from the underlying quantum symmetry algebra. Finally, notice that $\Upsilon_{A} \sim s^{4}$ in conjunction with the above scaling relations implies $v=1$. Thus, the upshot is that the anomalous fractional algebraic dynamical exponent $z=3 / 2$ is deeply rooted in the fusion relations for the quantum characters. This case establishes the superuniversality of superdiffusion with the dynamical exponent $z=3 / 2$ and rules out the possibility of different exponents such as $z=5 / 3$, which was suggested in Ref. [52] from a numerical analysis of SU (4) integrable chains. We will confirm this prediction numerically in the next section.

\section{NUMERICAL ANALYSIS}

We verify our predictions for the superdiffusive charge transport on a number of representative instances of integrable quantum spin chains. In our analysis, we include representative examples of quantum chains invariant under simple Lie groups from the classical series. Specifically, we consider homogeneous integrable spin-chain Hamiltonians whose local degrees of freedom transform in the fundamental representation of various symmetry groups (see Supplemental Material [58] for explicit expressions). Exceptional algebras are exempted from our numerical analysis. Moreover, we leave out the $B$ series, as the fundamental integrable $B_{2} \equiv \mathrm{SO}(5)$ chain has already been studied numerically in a recent paper [37].

We employ numerical tensor-network-based computations of the dynamical correlation function $C^{i i}(x, t)$ [as defined in Eq. (1)] in the canonical Gibbs equilibrium at infinite temperature. Owing to the non-Abelian symmetry $G$ of both the Hamiltonian $H$ and infinite-temperature Gibbs density matrix, all the components $i=1,2, \ldots, r$ are 
identical, and we thus suppress the redundant index $i$. We perform the time-evolving block-decimation (TEBD) algorithm in the Heisenberg picture [123-125], evolving the local charge density operator $q(0, t)$ in a matrix product operator (MPO) representation.

Numerical methods. In the simulations, a fourth-order Trotter decomposition is used to propagate the operator forward in time steps of size $\delta t$, followed by truncations at each bond keeping the largest $\chi$ Schmidt states. The simulations are accelerated by taking advantage of timereversal symmetry and translation symmetry to compute the full correlator $C(x, t)=\langle q(x, t / 2) q(0,-t / 2)\rangle$ using a single MPO evolution; additionally, the TEBD implements the maximal Abelian subgroup of the on-site symmetry group for efficiency. The computations are checked for convergence in $\delta t$ and bond dimension $\chi$ (up to $\chi=1024$ ).

Our TEBD scheme uses a Trotter step size of $\delta t=0.4$. The operators are truncated initially with a constant discarded weight $\varepsilon=10^{-8}$, allowing the bond dimension $\chi$ to grow until it reaches a threshold $\chi_{\max }=512$. Subsequent truncations keep only $\chi_{\max }$ states. The simulations are checked for convergence in the step size $\delta t$, the truncation error $\varepsilon$, and the threshold bond dimension $\chi_{\max }$. Additionally, the results shown here include rescaling of the correlations at each time to explicitly enforce the charge sum rule $\int d x C(x, t)=C(0,0)$, which improves the convergence significantly.

Results. Our main results are succinctly summarized in Fig. 1, where we display the dynamical width of the charge profiles

$$
[\Delta x(t)]^{2}=\frac{\int d x x^{2} C(x, t)}{\int d x C(x, t)} .
$$

We find a clear signature of an asymptotic power-law growth $\Delta x(t) \propto t^{1 / z}$, and the return probability shows powerlaw decay $C(0, t) \propto t^{-1 / z}$, with a numerically estimated dynamical exponent $z=3 / 2$ with great accuracy. The scaling collapse in Fig. 2 shows that the correlators obey the asymptotic scaling form $C(x, t) \simeq t^{-1 / z} f_{\mathrm{sc}}\left(x t^{-1 / z}\right)$. Comparing $f_{\mathrm{sc}}$ to the KPZ scaling function $f_{\mathrm{KPZ}}$ given by Eq. (1), we find some discernible deviations in the tails [including the basic $\mathrm{SU}(2)$ case studied previously in Ref. [30]]. On accessible timescales, we are unable to determine whether this discrepancy persists at late times or if it is merely due to transient effects.

\section{SEMICLASSICAL SPECTRUM}

The basic picture that we have established thus far is that the anomalous charge transport comes from giant quasiparticles (i.e., bound states carrying large bare Noether charge) immersed in a charge-neutral thermal background. It is natural to expect such giant quasiparticles to have a semiclassical description. In this section, we discuss such a semiclassical description. It is helpful to first review some results along these lines that were shown for $\mathrm{SO}(3)$ spin chains [44]. We then proceed by outlining how these arguments generalize to other symmetry groups.

Consider a one-dimensional classical SO(3) ferromagnet in its ground state, i.e., all spins aligned in some direction. The elementary excitations of the ferromagnet are soft Goldstone modes in which the spin orientation changes slowly across the lattice. One can regard any sufficiently slowly varying spin texture as being composed of Goldstone modes, as it locally consists of slow modulations (rotations) of the vacuum orientation. The long-wavelength dynamics of these ferromagnetic, quadratically dispersing, Goldstone modes is governed by a linear PDE. Away from the ground state, the evolution of the order parameter at large scales is governed instead by the nonlinear LandauLifshitz equation. Remarkably, the latter is an integrable PDE [126] that features stable, nonlinear, ballistically propagating modes of any width $w$ [127], known as solitons. The properties of these soliton solutions can be inferred by observing that they are wave packets of Goldstone modes that are stabilized by nonlinearity. For example, a soliton of width $w$ is made up of Goldstone modes with characteristic momentum $1 / w$ and thus characteristic energy density $1 / w^{2}$; therefore, its characteristic energy scales as $1 / w$ and its velocity also as $1 / w$. On the other hand, its spin orientation is away from the vacuum by $\mathcal{O}(1)$, so it carries a finite amount of magnetization, about $w$. These "bare" properties of classical solitons precisely match those of the large- $s$ Bethe ansatz strings, suggesting that there is some correspondence between them. Indeed, the exact correspondence between large- $s$ strings and large$w$ solitons can be explicitly established, not only at the level of individual spin-wave configurations as usual but even in high-temperature equilibrium ensembles [44]. (Note that, for this correspondence to hold, it is irrelevant whether the microscopic model has ferromagnetic or antiferromagnetic couplings: for the considerations in this paper, the Bethe vacuum always corresponds to the ferromagnetic ground state, and elementary excitations like solitons are always constructed above this state.)

This classical quasiparticle construction generalizes to arbitrary quantum or classical ferromagnets. As discussed below, distinguished common features of such ferromagnets are quadratically dispersing Goldstone modes. One can analogously construct slowly varying wave packets of these Goldstone modes; by construction, these have the same scaling as the large-s strings, so it is tempting to identify the two types of excitations in the general case also. Although we have not yet attempted to analytically solve the classical problem in full generality, by noting that large- $s$ strings must correspond to objects in the semiclassical spectrum and that Goldstone-like excitations exhaust this spectrum, it is tempting to infer the existence of such solitons from the integrability of the quantum model. 
However, one major puzzle arises when one tries to perform such an identification: The number of magnon flavors (which always coincides with the rank $r$ of the group $G$ ) can be far fewer than that of Goldstone modes; the latter is given by one-half of the real dimension of a coset manifold $G / H$, where $H$ is the residual symmetry group of the ferromagnetic state. There are apparently some "missing" Goldstone bosons that must, in some fashion, emerge out of the Bethe ansatz spectrum; however, they cannot be identified directly with elementary (physical and auxiliary) magnons of integrable quantum chains. Curiously, they rather correspond to "stacks" of magnons, which are longlived wave packets in a finite system, and only become sharp eigenstates in the infinite system limit.

The main result of this section is identifying the correspondence between Goldstone modes in the semiclassical spectrum and stacks of magnons (and condensates thereof, representing semiclassical Bethe $s$ strings) in the Bethe ansatz spectrum. Using this correspondence, one can identify large-spin semiclassical excitations above the ferromagnetic vacuum precisely as stacked strings. These semiclassical excitations can then be identified as solitons that involve smooth maps from $\mathbb{R}$ to the coset space $G / H$. While it would be interesting to construct such solitons directly in the classical theory, this task is left to future work.

\section{A. Counting nonrelativistic Goldstone modes}

Before detailing the spectra of classical continuous ferromagnets, we begin by first characterizing the spectrum of linear fluctuations of a ferromagnetic order parameter. This approach leads to the notion of Goldstone excitations, which naturally arise in systems with spontaneously broken, continuous internal symmetry. To this end, we briefly revisit the Goldstone theorem in a general context of nonrelativistic field theories. Our primary objective here is to characterize the (nonrelativistic) Goldstone modes that govern the low-energy spectrum for the class of integrable ferromagnetic quantum spin chains discussed in this work.

Let us first recapitulate the main result on the counting of Goldstone modes in systems without Lorentz invariance, as independently worked out in Refs. [128-130] (improving upon the earlier Nielsen-Chadha inequality [131]). The conventional setting concerns Hamiltonian systems invariant under a non-Abelian Lie group $G$, possessing a degenerate ferromagnetic ground-state manifold. Spontaneous breaking of internal symmetry amounts to picking a particular vacuum polarization (order parameter), say $\Omega$, thereby breaking the symmetry of the isometry group $G$ down to the stability subgroup $H \subset G$ of $\Omega$, determined by the condition

$$
h \Omega h^{-1}=\Omega, \quad h \in H .
$$

We are interested specifically in homogeneous (i.e., translation-invariant) ferromagnetic chains (of length $L$ ), where the global (pseudo)vacuum is simply a product state $\Omega_{L}=\bigotimes_{\ell=1}^{L} \Omega$. It is therefore sufficient to carry out the analysis at the level of local Hilbert spaces, and (with no loss of generality) we can assume $\Omega \equiv|0\rangle\langle 0|$.

At the algebraic level, the symmetry-breaking pattern $G \rightarrow H$ implies the following splitting of the generators, $\mathfrak{g}=\mathfrak{h} \oplus \mathfrak{m}$, with subalgebra $\mathfrak{h}$ constituting the generators of $H$ (which contains the Cartan subalgebra $\mathfrak{t}$ ) and $\mathfrak{m}$ denoting the linear span of the "broken generators" (these do not enclose an algebra). The number of broken generators is

$$
n_{b}=\operatorname{dim} \mathfrak{m}=\operatorname{dim}(G)-\operatorname{dim}(H) .
$$

Let $\mathrm{X}^{\sigma} \in \mathfrak{m}, \sigma \in\{1,2, \ldots, \operatorname{dim}(G / H)\}$ be a Hermitian basis of $\mathfrak{m}$. By the Goldstone counting theorem, the number of independent Goldstone modes $n_{\mathrm{GB}}$ is

$$
n_{\mathrm{G}}=n_{b}-\frac{1}{2} \operatorname{rank} \mathbf{V}
$$

with matrix

$$
\mathbf{V}_{a b}=-\mathrm{i}\left\langle 0\left|\left[\mathrm{X}^{a}, \mathrm{X}^{b}\right]\right| 0\right\rangle
$$

containing the vacuum expectation values of the commutators amongst the broken generators $X^{\sigma}$.

Goldstone modes come in two varieties, depending on the type of dispersion law at long wavelengths $(k \rightarrow 0)$, $\omega(k) \sim k^{\nu}$ : type I (or type $A$ ) with $\nu$ odd and type II (or type $B)$ with $\nu$ even. In general, we thus have

$$
n_{\mathrm{G}}=n_{\mathrm{I}}+n_{\mathrm{II}},
$$

with

$$
n_{\mathrm{I}}=n_{b}-2 n_{\mathrm{II}}, \quad n_{\mathrm{II}}=\frac{1}{2} \operatorname{rank} \mathbf{V} .
$$

In relativistic systems, Lorentz invariance forces $\mathbf{V}$ to be identically zero. In contrast, target spaces of nonrelativistic ferromagnets are symplectic manifolds. They have even (real) dimension, and consequently, $\mathbf{V}$ has full rank. Therefore, $n_{\mathrm{I}}=0$ and

$$
n_{\mathrm{G}} \equiv n_{\mathrm{II}}=\frac{1}{2} n_{b}
$$

In simple terms, every canonically conjugate pair of broken generators contributes an independent magnon branch, a quadratically dispersing, long-wavelength, spin-wave excitation of the ferromagnetic order parameter. 
TABLE I. Target spaces associated with classical continuous ferromagnets, describing the low-energy sector of quantum ferromagnetic chains with local degrees of freedom realized in the defining representation $\mathcal{V}_{\omega_{1}}$ of a Lie algebras $\mathfrak{g}$. Here, $n_{G}$ is the number of Goldstone modes in the spectrum.

\begin{tabular}{lccc}
\hline \hline $\mathfrak{g}$ & $\mathcal{V}_{\omega_{1}}$ & Target space $G / H$ & $n_{\mathrm{G}}$ \\
\hline$A_{n}$ & $(\mathbf{n})$ & $\mathrm{SU}(n) / \mathrm{U}(1) \times \mathrm{SU}(n-1)$ & $n-1$ \\
$B_{n}$ & $(\mathbf{2 n}+\mathbf{1})$ & $\mathrm{SO}(2 n+1) / \mathrm{U}(1) \times \mathrm{SO}(2 n-1)$ & $2 n-1$ \\
$C_{n}$ & $(\mathbf{2 n})$ & $\mathrm{USp}(2 n) / \mathrm{U}(1) \times \mathrm{USp}(2 n-2)$ & $2 n-1$ \\
$D_{n}$ & $(\mathbf{2 n})$ & $\mathrm{SO}(2 n) / \mathrm{U}(1) \times \mathrm{SO}(2 n-2)$ & $2 n-2$ \\
\hline \hline
\end{tabular}

\section{B. Symmetry-breaking patterns}

We now describe the spectra of Goldstone modes for the class of $G$-invariant ferromagnetic chains. The structure of coset spaces depends on the choice of a finite-dimensional irreducible on-site representation $\mathcal{V}_{\Lambda}$. Let us, mostly for simplicity, suppose for the moment that the local degrees of freedom transform in the defining representation of $\mathfrak{g}$. The highest weight is the first fundamental weight $\Lambda=\omega_{1}=$ $[1,0, \ldots, 0]$ of $\mathfrak{g}$, and the stabilizer $H \subset G$ of the vacuum state $\Omega$ has the structure $\mathrm{U}(1) \times H^{\prime}$ (i.e., $H$ is not semisimple). For the classical series, the coset structure is summarized in Table I.

Next, we specialize to the family of fundamental representations with $\Lambda=\omega_{a}$. In this case, the symmetrybreaking pattern can be inferred directly from the enumerated Dynkin diagrams with the use of a graphical recipe by simply "breaking the bonds" attached to the node with the nonzero label. This case is illustrated in Fig. 3 on two simple examples. The list of possibilities for all the classical series is summarized in Table II, with concrete examples provided in Table III. Employing the same trick allows us to also determine the structure of coset spaces for $G$-invariant classical ferromagnets corresponding to the exceptional groups $G_{2}, F_{4}, E_{6-8}$, specializing to the fundamental representations of $\mathfrak{g}$ (for completeness, we include them in the Supplemental Material).

The outlined recipe likewise applies for generic (i.e., nonfundamental), finite-dimensional, irreducible representations $\mathcal{V}_{\Lambda}$ of $\mathfrak{g}$ with Dynkin labels $\Lambda=\left[\omega_{1}, \ldots, \omega_{r}\right]$; the rule is to break all the bonds connecting to nodes with nonzero Dynkin labels. The resulting coset spaces are generalized flag manifolds $\mathcal{F}_{i_{1}, i_{2}, \ldots, i_{k}} \equiv G / H_{i_{1}, i_{2}, \ldots i_{k}}$, where the indices $i_{i}$ mark the nodes of the Dynkin diagram of $\mathfrak{g}$ with nonvanishing Dynkin labels (that is, $i_{i}=1$ if and only if $m_{i} \neq 0$ ). Compact complex manifolds $\mathcal{F}_{i_{1}, i_{2}, \ldots, i_{k}}$ indeed exhaust all Kähler manifolds (i.e., possess compatible Riemannian metric and symplectic structures), which are homogeneous spaces with a transitive action of $G$. Furthermore, points on such flag manifolds are in oneto-one correspondence with generalized coherent states in a specific representation of group $G$, namely,

$$
|\psi\rangle=\exp \left[\sum_{\alpha \in \Delta_{+}} w_{\alpha}^{-} \mathrm{E}^{-\alpha}\right]|\Lambda\rangle,
$$

for the highest weight state $|\Lambda\rangle$ of $\mathcal{V}_{\Lambda}$. However, if some $m_{a} \neq 0$, not all coordinates $w_{\alpha}^{-}$are independent, and some can be eliminated. For instance, in the unitary case ( $A$ series), the family of generalized flag manifolds has the structure

$\mathrm{SU}(n) / \mathrm{S}\left[\mathrm{U}\left(n_{1}\right) \times \mathrm{U}\left(n_{2}\right) \times \cdots \times \mathrm{U}\left(n_{j}\right) \times \mathrm{U}(1)^{\times k}\right]$,

for integers $j, k \geq 1$ and $n_{1} \geq n_{2} \geq \ldots \geq n_{j}>1$, subjected to $k+\sum_{i=1}^{j} n_{i}=n$. For example, for the case of $G=$ $\mathrm{SU}(3)$, there are two possible isotropy groups: $H_{1,0}=$ $H_{0,1}=\mathrm{U}(2)$ [as, e.g., for $(\mathbf{3}),(\overline{\mathbf{3}})$, or $(\mathbf{6})$ ] or $H_{1,1}=\mathrm{U}(1)^{\times 2}$ [as, e.g., for $(\mathbf{8}),(\mathbf{1 5})$, or $(\mathbf{2 7})$ ]. The upshot of that is that for quantum spin chains with different local degrees of

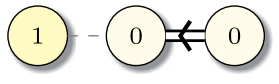

$\mathfrak{u}(1) \oplus \mathfrak{s p}(2)$

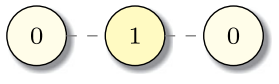

$\mathfrak{s u}(2) \oplus \mathfrak{u}(1) \oplus \mathfrak{s u}(2)$

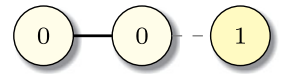

$\mathfrak{s u}(3) \oplus \mathfrak{u}(1)$

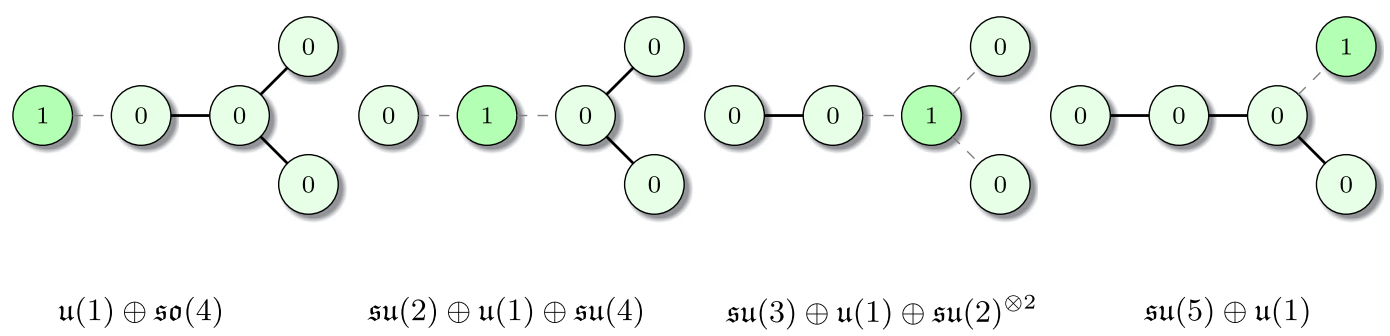

FIG. 3. Various symmetry-breaking patterns in fundamental ferromagnets with on-site irreducible representations $\mathcal{V}_{\omega_{a}}$, depicted for Lie algebras $C_{4}=\mathfrak{g p}(4)$ (top) and $D_{5}=\mathfrak{g} \mathfrak{o}(10)$ (bottom). The vacuum stability subgroup $H$ always includes a U(1) factor. The $n$th Dynkin node with label 1 corresponds to the $a$ th fundamental irreducible representation with highest weight $\Lambda=\omega_{a}$. Dashed lines designate the broken bonds. 
TABLE II. Symmetry-breaking patterns for all the fundamental representations $\Lambda=\omega_{k}$ for the family of classical Lie algebras $\mathfrak{g}$.

\begin{tabular}{lccc}
\hline \hline $\mathfrak{g}$ & irrep $\Lambda$ & Stabilizer $H$ & $\operatorname{dim}(G / H)$ \\
\hline$A_{n}$ & $\omega_{k}$ & $A_{k-1} \times \mathrm{U}(1) \times A_{n-k}$ & $2 k(n+1-k)$ \\
$B_{n}$ & $\omega_{k \leq n-2}$ & $A_{k-1} \times \mathrm{U}(1) \times B_{n-k}$ & $k(4 n+1-3 k)$ \\
$B_{n}$ & $\omega_{n-1}$ & $A_{n-2} \times \mathrm{U}(1) \times A_{1}$ & $(n+4)(n-1)$ \\
$B_{n}$ & $\omega_{n}$ & $A_{n-1} \times \mathrm{U}(1)$ & $n(n+1)$ \\
$C_{n}$ & $\omega_{k \leq n-2}$ & $A_{k-1} \times \mathrm{U}(1) \times C_{n-k}$ & $k(4 n+1-3 k)$ \\
$C_{n}$ & $\omega_{n-1}$ & $A_{n-2} \times \mathrm{U}(1) \times A_{1}$ & $(n+4)(n-1)$ \\
$C_{n}$ & $\omega_{n}$ & $A_{n-1} \times \mathrm{U}(1)$ & $n(n+1)$ \\
$D_{n}$ & $\omega_{k \leq n-3}$ & $A_{k-1} \times \mathrm{U}(1) \times C_{n-k}$ & $k(4 n-1-3 k)$ \\
$D_{n}$ & $\omega_{n-2}$ & $A_{n-3} \times \mathrm{U}(1) \times A_{1} \times A_{1}$ & $(n+5)(n-2)$ \\
$D_{n}$ & $\omega_{n-1}, \omega_{n}$ & $A_{n-1} \times \mathrm{U}(1)$ & $n(n-1)$ \\
\hline \hline
\end{tabular}

TABLE III. Coset spaces for all the fundamental representations $\Lambda=\omega_{a}$ for classical Lie algebras of rank 4 .

\begin{tabular}{cccc}
\hline \hline $\mathfrak{g}$ & On-site irrep & Target space $G / H$ & $n_{\mathrm{G}}$ \\
\hline$A_{4}$ & $(\mathbf{5}),(\overline{\mathbf{5}})$ & $\mathrm{SU}(5) / \mathrm{U}(1) \times \mathrm{SU}(4)$ & 4 \\
& $(\mathbf{1 0}),(\overline{\mathbf{1 0}})$ & $\mathrm{SU}(5) / \mathrm{U}(1) \times \mathrm{SU}(2) \times \mathrm{SU}(3)$ & 6 \\
$B_{4}$ & $(\mathbf{9})$ & $\mathrm{SO}(9) / \mathrm{SO}(2) \times \mathrm{SO}(7)$ & 7 \\
& $(\mathbf{3 6})$ & $\mathrm{SO}(9) / \mathrm{U}(1) \times \mathrm{SU}(2) \times \mathrm{SO}(5)$ & 11 \\
& $(\mathbf{8 4})$ & $\mathrm{SO}(9) / \mathrm{U}(1) \times \mathrm{SU}(2) \times \mathrm{SU}(3)$ & 12 \\
& $(\mathbf{1 6})$ & $\mathrm{SO}(9) / \mathrm{U}(1) \times \mathrm{SU}(4)$ & 10 \\
$C_{4}$ & $(\mathbf{8})$ & $\mathrm{USp}(8) / \mathrm{SO}(2) \times \mathrm{USp}(6)$ & 7 \\
& $(\mathbf{2 7})$ & $\mathrm{USp}(8) / \mathrm{U}(1) \times \mathrm{SU}(2) \times \mathrm{SO}(5)$ & 11 \\
& $(\mathbf{4 8})$ & $\mathrm{USp}(8) / \mathrm{U}(1) \times \mathrm{SU}(2) \times \mathrm{SU}(3)$ & 12 \\
& $(\mathbf{4 2})$ & $\mathrm{USp}(8) / \mathrm{U}(1) \times \mathrm{SU}(4)$ & 10 \\
$D_{4}$ & $\left(\mathbf{8}_{v}\right),(\mathbf{8}),\left(\mathbf{8}_{s}\right)$ & $\mathrm{SO}(8) / \mathrm{U}(1) \times \mathrm{SU}(3)$ & 6 \\
& $(\mathbf{2 8})$ & $\mathrm{SO}(8) / \mathrm{U}(1) \times \mathrm{SU}(2) \times 3$ & 9 \\
\hline \hline
\end{tabular}

freedom (i.e., transforming in different irreducible representations), the classical degrees of freedom of their emergent, effective, low-energy theories can live on the same target coset manifold. Below, we explain this fact from another perspective by directly examining the structure of the semiclassical spectrum.

Hermitian symmetric spaces. An important subclass of flag manifolds includes irreducible compact Hermitian symmetric spaces. Amongst cosets (42), one finds (in the special cases of $\Lambda=\omega_{a}$ ) complex Grassmannian manifolds $\mathrm{SU}(n) / \mathrm{S}[\mathrm{U}(k) \times \mathrm{U}(n-k)]$. The remaining infinite families of classical (i.e., nonexceptional) Hermitian symmetric spaces comprise (i) Lagrangian Grassmannians $\mathrm{USp}(2 n) / \mathrm{U}(n)$, associated with representations $\Lambda=\omega_{n}=$ $[0,0, \ldots, 1]$ from the $C_{n}$ series, $\mathrm{SO}(2 n) / \mathrm{U}(n)$ of complex dimension $\frac{1}{2} n(n-1)$, obtained from the fundamental spinor representations of $D_{n}$ (with Dynkin labels $\Lambda \in\left\{\omega_{n-1}, \omega_{n}\right\}$ ), and (ii) orthogonal Grassmannians $\mathrm{SO}(n) /[\mathrm{SO}(n-2) \times$ $\mathrm{SO}(2)$ ] of real dimension $2 n$, obtained from the defining $\mathrm{SO}(n)$ representations $\Lambda=\omega_{1}$ of $B_{n}$ or $D_{n}$ [notice, however, that compact Riemannian symmetric spaces, such as, e.g., $\mathrm{SU}(n) / \mathrm{SO}(n)$ and $\mathrm{SU}(2 n) / \mathrm{USp}(2 n)$, do not appear]. Concerning charge transport, we note that a recent numerical study [42] demonstrated that a class of integrable, nonrelativistic, classical sigma models (of the Landau-Lifshitz type) on complex and Lagrangian Grassmannians supports anomalous charge transport with the dynamical exponent $z=3 / 2$ (and KPZ scaling profiles).

\section{Goldstone modes in the Bethe-ansatz spectrum}

So far in this section, we have shown how to count Goldstone modes for a wide class of ferromagnets. These arguments do not rely on integrability. However, when the model is integrable, one can express all eigenstates using the Bethe ansatz, so the Goldstone modes must correspond to some type of object in the Bethe-ansatz spectrum. For the $\mathrm{SU}(2)$ case, the correspondence is obvious: the Bethe ansatz magnons are precisely the expected quadratic Goldstone modes. One might be tempted to conjecture that the same correspondence holds for other symmetry groups, namely, that the total number of internal degrees of freedom in generic classical field configurations will exactly match the number of distinct flavors of the Bethe roots, that is, $r=\operatorname{rank}(\mathfrak{g})$. However, one can quickly realize that there has to be a flaw in such a naive identification. There are two apparent inconsistencies that arise:

(1) First, consider the fundamental $\mathrm{SU}(n)$ ferromagnetic chains. For these chains, the Goldstone theorem predicts $n-1$ magnonic branches, and indeed, this number precisely agrees with the rank of $\mathrm{SU}(n)$, which also coincides with the total number of flavors in the $\mathrm{SU}(n)$ magnets. Nevertheless, one cannot directly identify the magnon branches with Goldstone branches since all but one of the magnon branches are auxiliary quasiparticles. In generic, excited, quantum eigenstates, auxiliary quasiparticles cannot be excited on their own without first having physical (i.e., momentum-carrying) excitations in a state. (By contrast, all the Goldstone branches are manifestly physical.)

(2) Consider, next, as another example, the $\mathrm{SO}(5)$ chain (which has rank $r=2$ ) made out of the fundamental degrees of freedom. The low-energy theory is associated with the coset space $\mathrm{SO}(5) /[\mathrm{SO}(2) \times \mathrm{SO}(3)]$, which is a real Grassmannian manifold of real dimension 6. Here, we expect to find three Goldstone modes, which now obviously exceeds the total number of flavors. More generally, one would expect the number of distinct types of macroscopic semiclassical strings to always equal $r=\operatorname{rank}(\mathfrak{g})$, which is typically vastly smaller than the number of independent Goldstone modes (cf. Sec. IV B).

To evade an apparent paradox, the only viable option is to look for additional degrees of freedom. We illustrate the resolution of this paradox first in the simplest example of 
SU(3) magnets, in an elementary way, and then extend our analysis to the general case in the following section.

\section{Example: $S U(3)$ ferromagnet}

In the SU(3) ferromagnet, there are two Goldstone modes. The single-site Hilbert space has three states, which we label $|0\rangle,|1\rangle,|2\rangle$. We choose our ferromagnetic ground state so that all spins are polarized in state 0 , i.e., $|\mathrm{GS}\rangle=\Omega_{L}=|00 \ldots 00\rangle$. Recall that the Hamiltonian simply permutes the labels on neighboring sites, so one can identify the low-lying excitations above the ferromagnet by inspection. There are two excitation branches; they correspond, respectively, to Bloch states of the form $\left|M_{1 ; n}\right\rangle=$ $(1 / \sqrt{L})\left(|100 \ldots 0\rangle+e^{2 n i \pi / L}|010 \ldots 0\rangle+\ldots\right)$ and $\left|M_{2 ; n}\right\rangle=$ $(1 / \sqrt{L})\left(|20 \ldots 00\rangle+e^{2 n i \pi / L}|020 \ldots 0\rangle+\ldots\right)$ (with $n=1$, $\ldots, L-1$, excluding the zero-momentum states $n=0$ ). These branches are symmetry related but do not mix under the Hamiltonian, so they are both quadratically dispersing (in the large-system limit) with identical dispersion relations.

As we noted above, there are manifestly two orthogonal magnon branches, but only one physical magnon in the Bethe ansatz spectrum. The resolution to this apparent paradox is as follows: When a non-Abelian symmetry is present, only the highest-weight eigenstates are directly constructed by the Bethe ansatz. All the remaining eigenstates are descendants and can be reached from these by acting with appropriate lowering operators. In the present case, one of the magnons is a descendant of the other and therefore is not explicitly present in the Bethe ansatz enumeration.

We now turn to the next simplest states, which are those with two flipped spins. There are two families of these states, one in which both flipped spins have the same flavor, i.e., involve configurations of the form $|01010 \ldots 0\rangle$, and another in which they have different flavors, i.e., involve configurations of the form $|01020 \ldots 0\rangle$. The latter type of state is distinguished from the former (in the Bethe ansatz enumeration) by containing two physical magnons and one auxiliary magnon. It is important to note that neither the type-1 nor the type-2 magnon is a well-defined propagating object in this state, as the magnons can "exchange" their flavor index. In other words, the flavor is not "bound" to each quasiparticle but has its own autonomous dynamics. (This phenomenon is analogous to spin-charge separation in the Hubbard model.)

Now, suppose we have a very long spin chain that contains two excitations of this form. A typical configuration close to the ground state would be $|000 \ldots 00100 \ldots 00200 \ldots 000\rangle$, i.e., the type- 1 and type- 2 magnons would be so widely separated that they could be regarded as effectively independent objects. In this scenario, it is natural to think of the type-1 magnon as "just" a physical magnon, whereas the type-2 magnon is a composite object containing one physical and one auxiliary magnon, which propagate together. This is the basic intuition underlying the concept of stacks, which we will discuss in more generality in what follows.

\section{General Goldstone-stack correspondence}

Let us remind the reader that magnon modes arise as plane-wave solutions to linear partial differential equations. On the other hand, classical field theories are genuinely interacting systems that are governed by nonlinear equations of motion. The latter exhibit a vastly richer spectrum of solutions. As mentioned earlier, a hallmark feature of nonlinear, completely integrable PDEs are soliton solutions, referring to nonlinear, ballistically propagating, particlelike field configurations whose stability is ensured by integrability. In the isotropic ferromagnets considered in this work, magnetic solitons assume a nontrivial internal structure. Indeed, from the perspective of quantum spin chains, solitons emerge as certain macroscopic coherent superpositions of magnons. Such states represent highly excited semiclassical eigenstates in the low-energy spectrum of the model, and their time evolution is generated by an effective classical Hamiltonian. There are various ways to establish this fact; the most standard and direct route is to make use of the standard path-integral techniques to project the quantum many-body Hamiltonian onto the manifold of (generalized) coherent states $|\psi\rangle$, formally achieved by rotating the (pseudo)vacuum, $|\psi\rangle=g|\Lambda\rangle$, as prescribed by Eq. (41). This way one can deduce a classical evolution for the ferromagnetic order parameter that takes values on a quotient manifold $G / H$, where $H$ is the vacuum stability subgroup defined above in Eq. (34) (see also, e.g., Refs. [42,132,133]). Classical fields can thus be identified with coordinates of generalized (Perelomov) coherent states [134].

Every admissible classical solution is a particular longwavelength (i.e., low-momentum) solution of the Bethe ansatz equations with a macroscopically large number of quanta. This particular regime, often referred to as the "asymptotic Bethe ansatz," was first studied by Sutherland [48] and, subsequently, more thoroughly examined in Ref. [135]. Nevertheless, an exact identification with classical field solutions was only made precise afterwards in Ref. [136], which provided a prescription to construct the associated classical spectral curve (the characteristic equation associated with the classical monodromy matrix; see, e.g., Refs. $[137,138])$ by transcribing the asymptotic Bethe equations as a Riemann-Hilbert problem. This method permits us to describe and classify the nonlinear modes for the class of so-called finite-gap solutions [139].

In the remainder of this section, we explain how the spectrum of both linear and nonlinear modes of classical integrable ferromagnets arises out of the nested Bethe ansatz magnon spectra in integrable $G$-invariant ferromagnetic quantum chains. A complete characterization of classical algebraic curves that emerges in this picture is a technical matter that falls outside of the scope of this study, and we hence do not attempt to undertake it here. 
Rather, we focus here on a deceptively innocent problem of identifying the relevant emergent classical degrees of freedom. The task turns out to be quite subtle, and in order to resolve it, we need a basic description of the asymptotic Bethe ansatz.

\section{Asymptotic Bethe ansatz}

We begin by noting that every finite-volume (highestweight) Bethe eigenstate in a quantum chain can be uniquely resolved in terms of magnon excitations. Individual unbound magnons correspond to real-valued Bethe roots with rapidities $\theta \sim \mathcal{O}\left(L^{0}\right)$. As described earlier, complex-valued rapidities of constituent magnons, which are part of a bound state, take the form of Bethe strings. Semiclassical eigenstates in the spectrum belong to highly excited states with $\mathcal{O}(L)$ excitations and large rapidities $\theta \sim \mathcal{O}(L)$, which can be thought of as condensing into a finite number of coherent macroscopic modes. In this low-energy regime, magnon momenta are inversely proportional to the system size, $p(\theta) \sim \mathcal{O}(1 / L)$. By accordingly introducing rescaled rapidities $\lambda_{j}^{(a)}$ via $\theta_{j}^{(a)}=L \lambda_{j}^{(a)}$, the Bethe equations in the leading order $\mathcal{O}(L)$ assume the form

$$
2 \pi n_{j}^{(a)}+\frac{\hat{m}_{a}}{\lambda_{j}^{(a)}}=\sum_{b=1}^{r} \sum_{k=1, k \neq j}^{N_{b}} \frac{t_{a}^{-1} \mathcal{K}_{a b}}{\lambda_{j}^{(a)}-\lambda_{k}^{(b)}} .
$$

As usual, $n_{j}^{(a)}$ represent (integer) mode numbers due to the multivaluedness of the complex logarithm.

Classical nonlinear modes pertain to bound states made of $\quad N_{a} \sim \mathcal{O}(L)$ constituent low-momentum magons. Crucially, rapidities of two nearby magnons are separated by an amount $o(L)$; however, since $\theta \sim \mathcal{O}(L)$, magnons are not, in general, spaced equidistantly as in the Bethe strings with $\theta \sim \mathcal{O}(1)$. What generically happens is that, upon taking the large- $L$ limit, rapidities $\lambda_{j}^{(a)}$ distribute densely along certain one-dimensional disjoint segments (i.e., contours) $\mathcal{C}^{(a)}=\cup_{i} \mathcal{C}_{i}^{(a)}$ in the spectral $\lambda$ plane, described by some smooth (in general, nonuniform) densities $\varrho_{a}(\lambda)$ of Bethe roots $\lambda_{j}^{(a)}$ with support on $\mathcal{C}^{(a)}$. Such macroscopic low-energy configurations assume a purely classical description, appearing as interacting "nonlinear Fourier modes" of nonlinear classical field configurations. The long-wavelength spectrum of a classical field may, in addition, involve a continuous spectrum of small fluctuations, namely, modes that carry infinitesimal chargesthese are the aforementioned Goldstone modes.

\section{Classical degrees of freedom: Stacks}

As we discussed in the previous subsection, the counting of Goldstone modes does not match that of magnons but rather that of stacks. We now discuss these emergent objects in detail. To the best our knowledge, stack excitations were first encountered in Refs. [140-142] in the studies of gauge-string duality in certain unitary supergroups that underlie the superconformal Yang-Mills theory $[143,144]$. Other than that, there has not been any general or systematic discussion devoted to these emergent and inherently classical objects so far in the literature. We proceed by explaining how the notion of stacks naturally arises in the context of integrable quantum ferromagnets with continuously degenerate ground states.

In the most basic terms, stacks pertain to excitations with an internal multiflavor structure. They are produced by merging together a subset of Bethe roots of distinct flavors that share the same rapidity (not merely just the real part). As we already emphasized previously, in a quantum spin chain, all rapidities must be pairwise distinct, and hence such configurations are prohibited. However, this restriction gets lifted in the low-momentum scaling limit since the Bethe roots of different flavors are allowed to approach arbitrarily close to each other and eventually recombine into an independent mode. It is clear that, by such a composition of magnons, one will be able to produce a large number of stacks. But only those stacks that involve momentum-carrying roots show up as physical fluctuations, whereas all the other stacks made solely from auxiliary excitations are likewise called auxiliary. Moreover, similar to the elementary excitations, stacks also exert attractive forces on themselves and thus can grow into nonlinear modes that carry finite filling fractions and charge densities.

The upshot is that for the complete classification of classical modes, one requires additional mode numbers to properly account for the internal structure of all the admissible stacks. This extra piece of information, which can be thought of various polarization directions in which a classical configuration can vibrate, depends on both the symmetry algebra and on-site representation under consideration. For illustration, let us look at our main example of the fundamental $\mathrm{SU}(n)$ chains. The asymptotic Bethe ansatz equations [see Eq. (43)], in the continuum description, turn into an $n$-sheeted Riemann surface whose sheets are "stitched together" by finitely many (square-root-type) branch cuts (see, e.g., Refs. [136,137] for additional details). The standard choice is to place the cuts along the contours $\mathcal{C}_{i}^{(a)}$ on which the Bethe roots condense. Creating a single excitation, say, of flavor $a$, amounts to connecting the $a$ th and $(a+1)$ th sheets with an infinitesimal cut. On the other hand, exciting a stack, say, of type $(a, b)$, corresponds to joining two nonadjacent sheets of the surface indexed by $a$ and $b+1$. Equations (43) need to be appropriately amended to incorporate these extra stack excitations. In Fig. 4, we illustrate the process of stack formation on a four-sheeted Riemann surface associated with the asymptotic Bethe equations of the SU(4) quantum chain made out of fundamental spins (4). 


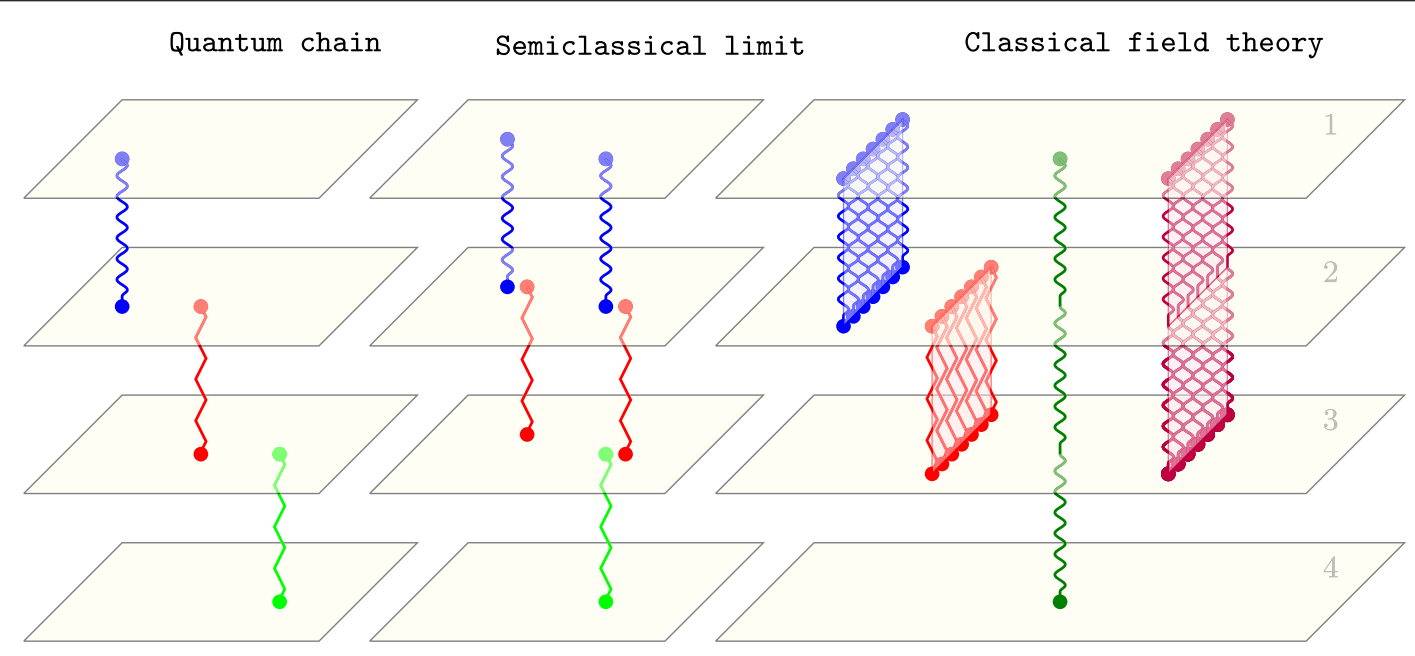

FIG. 4. Elementary and auxiliary excitations in the fundamental SU(4) quantum spin chain with on-site representation (4), represented on the associated four-sheeted Riemann surface. Left: physical momentum-carrying magnons (blue) emanating from the upper sheet, and two flavors of auxiliary magnons (red and green). Middle: semiclassical limit, depicting quantized stack excitations with nearly coinciding rapidities. Right: four-sheeted Riemann surface encoding the classical spectral curve of an effective low-energy field theory, with two types of excitations - isolated stacks, representing various branches of linear Goldstone modes, and condensates of stacks (square-root branch cuts) pertaining to nonlinear interacting modes carrying a finite amount of energy. Physical stacks (dark green) and condensates (blue and purple) involve momentum-carrying excitations. Condensates of stacks of a uniform density are identified with classical solitons.

In summary, the number of independent classical degrees of freedom equals the number of distinct physical stacks. We reemphasize that stack excitations are not permitted in the spectrum of a quantum chain due to kinematic constraints. In other words, upon including quantum fluctuations [even perturbatively at the level of asymptotic equations (43)] stacks immediately disintegrate into individual magnons.

In the next section, we describe how to determine the admissible symmetry-breaking patterns and classify the corresponding physical stacks.

\section{Giant quasiparticles as classical solitons}

In the previous section, we have not mentioned how soliton modes enter the picture. While classical solitons are, strictly speaking, not part of the nonlinear (finite-gap) spectrum, they nevertheless emerge as from elliptic waves in the limit of unit elliptic modulus. Thus, they are only made possible after decompactifying the spatial dimension $[136,145]$. By tracing this process in the spectral plane, one finds that solitons emerge out of a "singular event" in which two density contours close by merge with one another, resulting in a single, vertically oriented contour with constant unit density of Bethe roots. Such objects have been described before in certain integrable classical sigma models—see, e.g., Refs. [136,137,141,145]—where they are normally called condensates. It is important to stress, however, that these are distinct from ordinary condensates that constitute square-root branch cuts of finite-genus Riemann surfaces. From the viewpoint of an algebraic curve, such uniform condensates arise from coalescing two square-root branch cuts into a logarithmic branch cut. The giant quasiparticles from the preceding discussion, namely, bound-state excitations with a macroscopic number of quanta carrying low momenta, are therefore none other than quantized solitons.

\section{Counting stacks}

Now, we finally address the "counting problem." The remaining task is to extract the correct number of Goldstone

TABLE IV. Positive roots systems for Lie algebras $A_{3}=\mathfrak{g} \mathfrak{u}(4)$ and $B_{3}=\mathfrak{g} \mathfrak{p}(7)$ of rank 3 . In the $\alpha$ basis, any positive root $\alpha \in$ $\Delta_{+}$can be decomposed as an integral linear combination of simple roots $\alpha_{i}$ (level equals the sum of coefficients), whereas the $\omega$ basis provides an expansion in terms of the fundamental weights (with coefficients being the Dynkin labels).

\begin{tabular}{ccc}
\hline \hline$\alpha$ basis & $\omega$ basis & Level \\
\hline$(1,1,1)$ & {$[1,0,1]$} & 3 \\
$(0,1,1)$ & {$[-1,1,1]$} & 2 \\
$(1,1,0)$ & {$[1,1,-1]$} & 2 \\
$(0,0,1)$ & {$[0,-1,2]$} & 1 \\
$(0,1,0)$ & {$[-1,2,-1]$} & 1 \\
$(1,0,0)$ & {$[2,-1,0]$} & 1 \\
$(1,2,2)$ & {$[1,0,1]$} & 5 \\
$(1,1,2)$ & {$[1,-1,2]$} & 4 \\
$(0,1,2)$ & {$[-1,0,2]$} & 3 \\
$(1,1,1)$ & {$[1,0,0]$} & 3 \\
$(0,1,1)$ & {$[-1,1,0]$} & 2 \\
$(1,1,0)$ & {$[1,1,-2]$} & 2 \\
$(0,0,1)$ & {$[0,-1,2]$} & 1 \\
$(0,1,0)$ & {$[-1,2,-2]$} & 1 \\
$(1,0,0)$ & {$[2,-1,0]$} & 1 \\
\hline \hline
\end{tabular}


modes from the semiclassical spectrum of quantum ferromagnetic chains whose excitations, both physical and auxiliary, carry $r$ distinct flavor numbers (one per each simple root $\alpha_{i}$ of $\mathfrak{g}$ ). Different types of stacks can be generated simply by forming all linear combinations of simple roots $\alpha_{i}$ with non-negative integral coefficients, see Table IV for examples. In other words, excitation operators associated with stacks correspond to positive roots $\alpha \in \Delta_{+}(\mathfrak{g})$. The subset of physical roots corresponds to those roots that involve at least one physical simple root, associated with physical (i.e., momentum-carrying) excitations. In the fundamental models, for example, it is only $\alpha_{1}$.

By making use of partially ordered sets known as Hasse diagrams, we, in turn, describe a helpful graphical representation for all the physical stacks, as exemplified in Fig. 5. Let us first outline the construction by specializing to fundamental irreducible on-site representations $\Lambda=\omega_{a}$. We start by picking one of the bottom nodes (associated with simple roots) and proceed by 'climbing up' in the direction of the top node (the highest root) by successively adding one simple root at a time. With this rule, one can find several inequivalent paths through the Hasse diagram. Since each vertex is an independent Goldstone mode, their total number $n_{\mathrm{G}}$ equals the total number of vertices visited along the way. More generally, namely, for the nonfundamental irreducible on-site representations $\mathcal{V}_{\Lambda}$ with $\Lambda=\sum_{a=1}^{r} m_{a} \omega_{a}$ involving multiple nonvanishing Dynkin labels $m_{a}$, one finds multiple momentum-carrying species in the spectrum. In this case, one has to simply superimpose all the sublattices that emanate from base nodes $\alpha_{i}$ for all $a \in \mathcal{I}_{r}$ for which $m_{a} \neq 0$. The maximal breaking corresponds to all $m_{a}$ being nonzero (in which case, the isotropy group $H$ coincides with the maximal torus subgroup $T$ of $G$ ), resulting in the maximal number of Goldstone modes $n_{G}=\frac{1}{2} \operatorname{dim}(G / T)=\left|\Delta_{+}\right|$, rendering all stacks physical.

Finally, in cases when a Lie algebra $\mathfrak{g}$ involves a node of connectivity 3 (that is, types $D_{r}$ and $E$ families), the Hasse lattices extend into the third dimension [this is illustrated in Fig. 6 for the example of $\mathfrak{s o}(10)]$.
Hasse lattice of $\Delta_{+}$

$$
A_{3}=\mathfrak{s u}(4)
$$

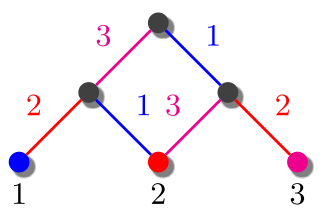

$B_{3}=\mathfrak{s o}(7)$

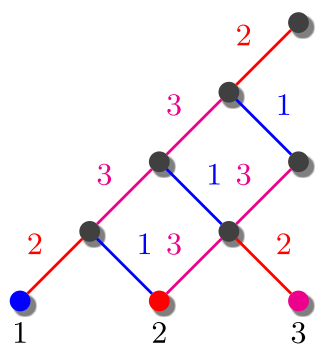

Fundamental irreps $\Lambda=\omega_{a}$

(4)

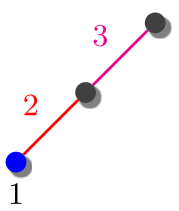

(7)

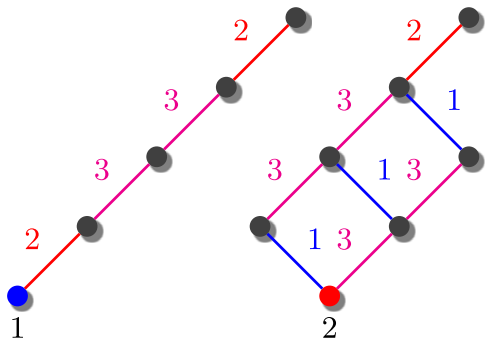

(6)

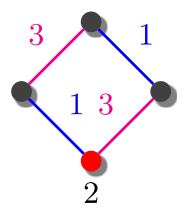

(21)

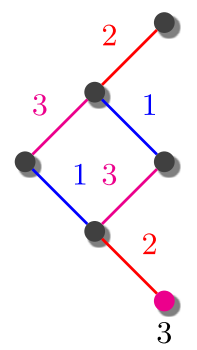

(8)

$(\overline{4})$

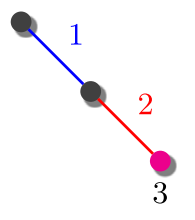

Nonfundamental irrep $\Lambda=[1,0,1]$

(15)

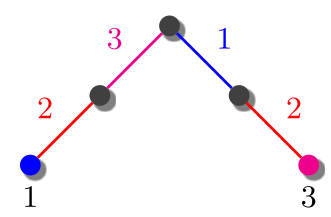

(48)

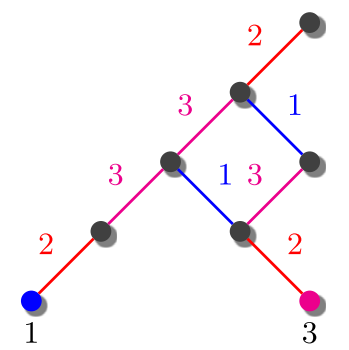

FIG. 5. Left: Hasse diagrams associated with the sublattices $\Delta_{+}$of positive roots of $\mathfrak{g}$, shown for Lie algebras $\mathfrak{g} \mathfrak{i t}(4)$ (top) and $\mathfrak{g} \mathfrak{p}(7)$ (bottom) of rank 3. The nodes represent positive roots $\alpha \in \Delta_{+}$, with the simple ones (at the bottom) marked with different colors. Moving upwards along a colored edge amounts to adding a simple root corresponding to that color. Positive roots are separated into $\ell$ levels, given by the sum of all positive integral coefficients in the expansion of $\alpha$ over the basis of simple roots $\alpha_{i}$. The total number of positive roots yields the maximal number of Goldstone modes $n_{G}=\left|\Delta_{+}\right|=\frac{1}{2} \operatorname{dim}(G / T)$ for the full breaking of symmetry $G \rightarrow T$. Middle: Hasse sublattices pertaining to all the distinct fundamental irreducible representations $\Lambda=\omega_{a}$ (i.e., Young diagrams with a single column), connecting the base node $\alpha_{a}$ to the upper node (maximal root). The total number of nodes in a given sublattice equals the number of distinct branches of magnon excitations (Goldstone bosons). Right: Hasse sublattices for nonfundamental on-site representations (shown for $\Lambda=[1,0,1]$ ) obtained by superimposition of the fundamental sublattices, each belonging to a single nonvanishing Dynkin label. 


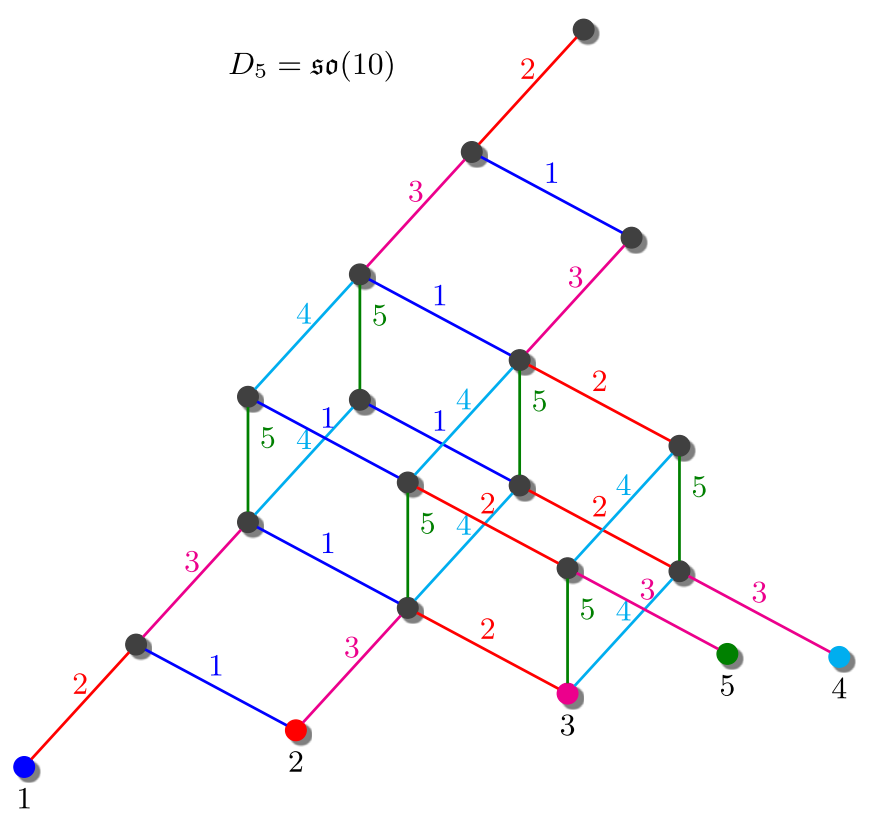

FIG. 6. Hasse diagram for the positive root lattice $\Delta_{+}(\mathfrak{g o}(10))$. There are 20 roots in total (black dots), arranged into 7 levels. The lattice extends into three dimensions as the simple root $\alpha_{3}$ has connectivity 3 . To find the $\alpha$ pattern corresponding to a simple root $\alpha_{i}$, one begins at $\alpha_{i}$ and also collects the nodes by always proceeding forward in the direction of the highest root. The $\mathbb{Z}_{2}$ automorphism of the Dynkin graph reveals itself as the reflection symmetry with respect to the main diagonal of the cube passing though nodes 4 and 5.

\section{CONCLUSION}

In this work, we initiated a systematic study of anomalous transport at finite temperature, which was recently uncovered in a number of quantum integrable systems. We aimed mainly at settling whether all integrable models with local and isotropic interactions-that, in addition, possess a global continuous symmetry of the non-Abelian Lie group-display superdiffusion of the Noether charges. By establishing the link between the thermodynamic quasiparticle content and representations of the corresponding quantum groups, we first obtained universal algebraic dressing equations and subsequently performed a scaling analysis of these dressing equations within the framework of generalized hydrodynamics. Our conclusion is that anomalous charge transport with a superdiffusive dynamical exponent $z=3 / 2$ is a common feature of all integrable ferromagnetic lattice models with short-range interactions, provided the Hamiltonian exhibits invariance under a nonAbelian continuous symmetry. This conclusion holds irrespective of the symmetry group or unitary irreducible representations associated with (local) physical degrees of freedom. Each such subclass indeed constitutes an infinitely large family of commuting integrable Hamiltonians, all of which exhibit $z=3 / 2$ superdiffusion. Moreover, we have argued that any other anomalous exponent is incompatible with the underlying quasiparticle structure. In addition, the same type of anomalous charge transport persists for group-valued Noether currents, even in Lorentz-invariant and nonrelativistic integrable quantum field theories. Because of its remarkable level of robustness, we dubbed this phenomenon as superuniversal.

Let us briefly restate the key steps that lead to this general conclusion. We begin by recalling that anomalous transport of some charge $Q$ occurs only when $Q$ is one of the generators of a non-Abelian subgroup $H \subseteq G$ such that the equilibrium state is invariant under $H$. If one considers generic polarized states (realized by applying chemical potentials to the Cartan charges), the anomalous conductivity gets regularized, and one recovers ballistic transport with subleading diffusive corrections, as one generically expects within generalized hydrodynamics. Upon approaching the $G$-symmetric state, however, one finds that the Drude weights governing the ballistic transport vanish, whereas the diffusion constants simultaneously diverge. In this regime, the quasiparticles that dominate the magnetic susceptibility and transport are macroscopically large, coherent bound states of magnons, which we call giant quasiparticles. All other nongiant quasiparticles become effectively depolarized and do not contribute either to transport or to susceptibility. Transport of the Noether charges is, in effect, described by a dense gas of interacting giant quasiparticles. Each of these giant quasiparticles moves with a characteristic velocity inversely proportional to its width: We established this result by explicit analysis of the GHD equations and interpreted it as indicating that these giant quasiparticles are solitons made up of nonlinearly interacting, quadratically dispersing Goldstone modes. When these modes are present at finite density, they dress each other's charge in a nonperturbative way, and this nontrivial dressing leads to the fractional dynamical exponent $z=3 / 2$.

To further elucidate the nature of the giant quasiparticles, we, in addition, examined the structure of semiclassical eigenstates from the viewpoint of an effective low-energy theory with respect to a (continuously degenerate) ferromagnetic pseudovacuum. At the level of an integrable classical field theory, giant quasiparticles can be identified with soliton modes. The latter may be thought of as the nonlinear counterparts of quadratically dispersing Goldstone bosons. As we explain, the number of internal degrees of freedom of classical soliton modes does not equal the number of distinct flavors of the elementary excitations that constitute the quasiparticle content of an integrable quantum chain, contrary to naive expectations. Instead, the total number of polarization directions of a classical field typically exceeds the rank of the group $G$. This mismatch is due to the formation of emergent classical multiflavored degrees of freedom called stacks, which are produced by gluing together magnons of different flavors (which dissolve upon introducing quantum corrections). 
Stacks that contain momentum-carrying magnons should be regarded as independent physical excitations, and we outlined how they can be naturally arranged on vertices of the Hasse diagrams. The total number of physical stacks is found to be in perfect agreement with the prediction of the Goldstone theorem. As a by-product of our work, we provided a full classification of ferromagnets with the global symmetry of a simple Lie algebra.

At the formal level, the anomalous nature of charge transport can be attributed to a particular structure of fusion identities amongst the characters of Yangian symmetry algebras, which enforces rigid algebraic constraints on the thermodynamic quasiparticle spectra. The list of models discussed in this study is therefore unlikely to be exhaustive, and other classes of integrable models not included in this study are likewise expected to feature a superdiffusive charge transport with characteristic exponent $z=3 / 2$. Specifically, we anticipate that the phenomenon extends beyond Yangians associated with simple Lie algebra to other classes of models that arise from rational solutions to the Yang-Baxter equations as, for example, the FermiHubbard model [38], and to other integrable spin chains that realize Yangian symmetries associated with unitary or orthosymplectic Lie superalgebras, that is, $\mathfrak{g} \mathfrak{t}(n \mid m)$ and $\mathfrak{o} \mathfrak{G} \mathfrak{p}(n \mid 2 m)$ (see, e.g., Refs. [79,146,147]), whose bosonic Noether charges are known to display singular diffusion constants [46].

To benchmark our predictions, we have performed numerical simulations on a handful of representative instances of integrable quantum chains, restricting the analysis to classical simple Lie algebras and only to fundamental local degrees of freedom. We confirmed the predicted anomalous algebraic dynamical exponent $z=$ $3 / 2$ with great numerical precision. Unfortunately, we could not reliably discern the anticipated KPZ scaling profiles. Quantum spin chains with the symmetry of exceptional Lie algebras have not been included in the present numerical study, largely for technical reasons. Since constructing integrable quantum chains based on the "quantized symmetry" of an exceptional Lie algebra is algebraically rather laborious (see, e.g., Ref. [148]), we prefer to leave them for a separate technical study. The other practical difficulty is that dimensions of the defining irreducible representations are, possibly with the exception of (7) and (14) of $G_{2}$, conceivably too large to be efficiently simulated with current DMRG techniques.

Finally, a central piece of the full puzzle is still missing: Despite integrability, a first-principles derivation of the KPZ asymptotic scaling profiles of dynamical structure factors remains elusive. At this time, there only exists a phenomenological picture, which, under certain plausible assumptions, justifies the emergence of a noisy Burgers equation for the basic $\mathrm{SU}(2)$ case [45]. One obvious drawback of such an approach is that it cannot yield quantitative predictions such as the value of the KPZ coupling. Understanding quantitatively, from $a b$ initio principles, how KPZ universality emerges (and not only $z=3 / 2$ ) remains a major challenge for future works, even in the case of the $\mathrm{SU}(2)$ symmetric Heisenberg $X X X$ spin chain. Another open problem is to appropriately extend the proposal of Ref. [44] to systems that are invariant under symmetries of higher rank in order to extract the KPZ nonlinearity constant.

\section{ACKNOWLEDGMENTS}

We thank Vir Bulchandani, Michele Fava, Žiga Krajnik, Fedor Levkovich-Maslyuk, Sid Parameswaran, Tomaž Prosen, and Marko Žnidarič for comments, stimulating discussions, and collaboration on related topics. This work was supported by the National Science Foundation under NSF Grant No. DMR-1653271 (S. G.), the US Department of Energy, Office of Science, Basic Energy Sciences, under Early Career Award No. DE-SC0019168 (R. V.), the Alfred P. Sloan Foundation through a Sloan Research Fellowship (R. V.), the Research Foundation Flanders (F. W. O. and J.D. N.), and the Slovenian Research Agency (ARRS) program No. P1-0402 (E. I.).

\section{APPENDIX A: DRESSED CHARGE FLUCTUATIONS}

For a general equilibrium macrostate characterized by quasiparticle densities $\rho_{A}(\theta)$, dressed charge susceptibilities (in the Cartan sector) can be linked to density fluctuations via

$$
\begin{aligned}
\left\langle q_{A}^{(i) \mathrm{dr}} q_{A}^{(j) \mathrm{dr}}\right\rangle & =\sum_{B, B^{\prime}} \int d \theta d \theta^{\prime} \frac{\delta q_{A}^{(i) \mathrm{dr}}}{\delta \rho_{B}(\theta)} \frac{\delta q_{A}^{(j) \mathrm{dr}}}{\delta \rho_{B^{\prime}}(\theta)} \\
& \times\left\langle\delta \rho_{B}(\theta) \delta \rho_{B^{\prime}}\left(\theta^{\prime}\right)\right\rangle .
\end{aligned}
$$

Variations of $q_{A}^{(i) \mathrm{dr}}$ with respect to quasiparticle densities $\rho_{A}(\theta)$ can be expressed as

$$
\frac{\delta q_{A}^{(i) \mathrm{dr}}}{\delta \rho_{B}(\theta)}=\sum_{B} K_{A B}^{\mathrm{dr}}(\theta) q_{B}^{(i) \mathrm{dr}},
$$

where $K_{A B}^{\mathrm{dr}}(\theta)$ are the dressed scattering differential phases. Using that [149]

$$
\left\langle\delta \rho_{A}(\theta) \delta \rho_{A^{\prime}}\left(\theta^{\prime}\right)\right\rangle=\frac{1}{\ell} C_{A A^{\prime}}\left(\theta, \theta^{\prime}\right),
$$

and employing, for convenience, the following compact matrix notations,

$$
\begin{gathered}
\mathbf{C}=\boldsymbol{\Omega}[\mathbf{n}]^{-1} \boldsymbol{\rho}(\mathbf{1}-\mathbf{n}) \boldsymbol{\Omega}[\mathbf{n}], \\
\mathbf{K}^{\mathrm{dr}}=\boldsymbol{\Omega}[\mathbf{n}] \mathbf{K},
\end{gathered}
$$


where $\boldsymbol{\Omega}[\mathbf{n}] \equiv(\mathbf{1}+\mathbf{K n})^{-1}$, we arrive at

$$
\left\langle q_{A}^{(i) \mathrm{dr}} q_{A}^{(j) \mathrm{dr}}\right\rangle=\frac{1}{\ell}\left(\mathbf{K}^{\mathrm{dr}} \mathbf{q}^{\mathrm{dr}} \mathbf{C} \mathbf{q}^{\mathrm{dr}} \mathbf{K}^{\mathrm{dr}}\right)_{A A},
$$

where $\mathbf{q}^{\mathrm{dr}}$ stands for the diagonal matrix of dressed charges $q_{A}^{\mathrm{dr}}$.

For the Cartan charges $Q^{(i)}$, in an unpolarized background (i.e., $h_{i}=0$ for all $i=1,2, \ldots, r$ ), we have $q_{a, s}^{(i) \mathrm{dr}}=0$, with an exception in the limit $s \rightarrow \infty$ where the above expression simplifies to give Eq. (25).

\section{APPENDIX B: ANALYTIC SOLUTIONS}

In order to infer the nature of charge transport in an unpolarized thermal ensemble, it is essential to deduce the asymptotic properties of the TBA state functions for the giant quasiparticles, namely, for the large Bethe strings in the $s \rightarrow \infty$ limit. To this end, we subsequently examine certain formal properties of the dressing equations for the specific algebraic case of the grand-canonical Gibbs equilibrium ensembles in the limit of infinite temperature. We study states with unbroken symmetry $G$, requiring us to switch all the U(1) chemical potentials off.

To keep our presentation succinct, we only provide the essential information. For more details and definitions, we refer the reader to the Supplemental Material [58].

\section{Asymptotic analysis}

We first carry an asymptotic analysis at large $s$ at a formal level.

Fermi functions. Integrable quantum chains that are associated with the class of evaluation representations of the Yangian algebra exhibit Fermi-Dirac statistics. The occupation function scales at large $s$ as $n_{a, s} \sim 1 / s^{2}$ (and, accordingly, $\mathcal{Y}_{a, s} \sim s^{2}$ ). This is a direct corollary of the Hirota relation (in this particular context, known also as the $T$ system) for the "quantum characters" $\mathcal{T}_{a, s}$, corresponding to the large- $\theta$ limits of the thermodynamic $\mathcal{T}$ functions; see Ref. [58] for details. The key property in this respect is that the $\mathcal{T}$ functions $\mathcal{T}_{a, s}$ are, by construction, polynomials in $s$ (for all $a \in \mathcal{I}_{r}$ ), which readily implies that the $\mathcal{Y}$ functions $\mathcal{Y}_{a, s}$ are rational functions that only depend on quantum numbers $(a, s)$.

State densities. The above scaling of $n_{a, s}$ immediately implies the asymptotic relation $\rho_{a, s}^{\text {tot }} \sim s^{2} \rho_{a, s}$. Notice that while both state densities $\rho_{a, s}(\theta)$ and $\rho_{a, s}^{\text {tot }}(\theta)$ likewise depend algebraically on $s$, in distinction to Fermi functions, they also depend nontrivially on $\theta$ (even in the limit of infinite temperature).

We are now in a position to analyze the large-s behavior of the dressing equations. We start with the simple case of the spin-1/2 Heisenberg chain SU(2), involving only one species of magnons. The analysis can be performed on the canonical form of the Bethe-Yang equations,

$$
\rho_{s}^{\mathrm{tot}}=K_{s}-\sum_{s^{\prime} \geq 1} K_{s, s^{\prime}} n_{s^{\prime}} \rho_{s^{\prime}}^{\mathrm{tot}}
$$

Two observations can readily be made: (i) For a fixed value of $\theta$, the kernels fall off as $K_{s}(\theta)=2 \pi p_{s}^{\prime}(\theta) \sim 1 / s$, and (ii) because of the telescopic cancellation of poles, the differential scattering phase shifts $K_{s, s^{\prime}}$ involve 2- $\min \left(s, s^{\prime}\right)$ terms. Thus, we can make the following estimate at large $s$ :

$$
\rho_{s}^{\mathrm{tot}} \lesssim \frac{1}{s}+\sum_{s^{\prime}=1}^{s} 2 s^{\prime} n_{s^{\prime}} \rho_{s^{\prime}}^{\mathrm{tot}}+2 s \sum_{s^{\prime}=s+1}^{\infty} n_{s^{\prime}} \rho_{s^{\prime}}^{\mathrm{tot}},
$$

where each term under the sum has been upper bounded by a constant using the sum rule $1 \star K_{s}=1$. The second sum can be understood as the residual contribution coming from large $s^{\prime}$ strings with $s^{\prime}>s$, which gets suppressed in the $\sim 1 / s$ fashion. The first sum converges in the $s \rightarrow \infty$ limit, provided $\lim _{s \rightarrow 0} \rho_{s}^{\text {tot }}=0$, when the total density of states $\rho_{s}^{\text {tot }}$ decays to zero at large $s$ (in an algebraic fashion). Lastly, using that the bulk recurrence relations pertaining to the algebraic dressing equations involve only rational functions of $s$, this implies $\rho_{s}^{\text {tot }} \sim 1 / s$ scaling at large $s$.

In more general integrable models (with nested spectra), one has to additionally account for quasiparticles of different flavors, whose mutual interactions are prescribed by the Cartan matrix $\mathcal{K}$ (cf. Ref. [58] for the definition). The above argument can be easily adapted by including an extra sum over the flavor index.

\section{Analytic solutions in the high-temperature limit}

To solidify our statements and conclusions, here we explicitly work out the exact closed-form solution to the algebraic TBA equations. The full solution for the basic cases of the $\mathrm{SU}(2)$ spin- $S$ (Babujian-Takhtajan) quantum chains has already been obtained in Ref. [109]. Here, we are primarily interested in models that possess Lie symmetries of higher rank.

For definiteness, we specialize to $\mathrm{SU}(n)$ quantum chains with fundamental on-site degrees of freedom. While other cases can be treated in a similar fashion, for compactness of presentation, we postpone a complete and comprehensive analysis for a separate technical study.

Let us consider, as our starting point, the algebraic limit of the group-theoretic dressing equations [58], which, in Fourier $k$ space, turn into a system of coupled algebraic equations of the form (with implicit summation over repeated indices)

$$
s^{-1} \star F_{a, s}-I_{s, s^{\prime}} \bar{n}_{a, s^{\prime}} F_{a, s^{\prime}}-I_{a, a^{\prime}} n_{a^{\prime}, s} F_{a^{\prime}, s}=\delta_{a, s},
$$

with $s^{-1}(k)=2 \cosh (k / 2)$ and adjacency matrix $I_{a, b}=$ $\delta_{a, b-1}+\delta_{a, b+1}$. The first step is to solve the homogeneous part, which can be achieved with the following ansatz:

$F_{a, s}(k)=A_{a, s}(k) K_{a+s-1}(k)+B_{a, s}(k) K_{a+s+1}(k)$, 
where $K_{s}(k)=e^{-s|k| / 2}$ are the elementary scattering kernels in Fourier space. Plugging this ansatz into Eq. (B3), performing the Laplace transform from the $k$ plane to the $z$ plane, and demanding the null condition for all the residues located at $z_{i} \in \frac{1}{2} \mathbb{N}$, we end up with the following system of recurrence relations:

$$
\begin{gathered}
A_{a, s}=\bar{n}_{a, s-1} A_{a, s-1}+n_{a-1, s} A_{a-1, s}, \\
B_{a, s}=\bar{n}_{a, s+1} B_{a, s+1}+n_{a+1, s} B_{a+1, s}, \\
A_{a, s}-B_{a, s}=\bar{n}_{a, s+1} A_{a, s-1}+n_{a+1, s} A_{a+1, s} \\
-\bar{n}_{a, s-1} B_{a, s-1}+n_{a-1, s} B_{a-1, s} .
\end{gathered}
$$

The Fermi functions can be conveniently expressed in terms of ratios of $\mathfrak{g} \mathfrak{u}(n)$ characters $\chi_{a, s}$,

$$
n_{a, s}=\frac{\chi_{a-1, s} \chi_{a+1, s}}{\chi_{a, s}^{2}}, \quad \bar{n}_{a, s}=\frac{\chi_{a, s-1} \chi_{a, s+1}}{\chi_{a, s}^{2}}
$$

(see Ref. [58] for additional information). The solution has the form

$$
\begin{aligned}
& A_{a, s}=A_{0} \frac{\chi_{a, s} \chi_{a-1, s-1}}{\chi_{a-1, s} \chi_{a, s-1}}, \\
& B_{a, s}=B_{0} \frac{\chi_{a, s} \chi_{a+1, s+1}}{\chi_{a+1, s} \chi_{a, s+1}},
\end{aligned}
$$

with coefficients $A_{0}$ and $B_{0}$ yet to be determined. By plugging these back into Eqs. (B7), we retrieve the Hirota relation for classical characters. To fix the undetermined constants, we find the particular solution of Eq. (B3), with the source term attached at the first node at $(a, s)=(1,1)$, yielding $A_{0}=-B_{0}=1 / \chi_{1,1}$. Transforming back to the $\theta$ plane, we finally obtain

$$
\begin{aligned}
F_{a, s}(\theta)= & \frac{\chi_{a, s}}{\chi_{1,1}}\left[\frac{\chi_{a-1, s-1}}{\chi_{a-1, s} \chi_{a, s-1}} K_{a+s-1}(\theta)\right. \\
& \left.-\frac{\chi_{a+1, s+1}}{\chi_{a+1, s} \chi_{a, s+1}} K_{a+s+1}(\theta)\right] .
\end{aligned}
$$

In the context of anomalous transport, we are solely interested in the $x_{i} \rightarrow 1$ limit. In this limit, the $\mathfrak{g} \mathfrak{t}(n)$ characters become the dimensions $d_{a, s}$ of rectangular irreducible representations $\mathcal{V}_{s \omega_{a}}$; more details are provided in Ref. [58]. Specifically, the total state densities read

$$
\rho_{a, s}^{\mathrm{tot}}(\theta)=\frac{s+n-a}{n}\left[\frac{K_{s+a-1}(\theta)}{s+a-1}-\frac{K_{s+a+1}(\theta)}{s+a+1}\right] .
$$

There is an analogous expression (up to a multiplicative prefactor) for the dressed differential quasiparticle energies $\varepsilon_{a, s}^{\prime}(\theta)$, which can be obtained by simply replacing $K_{s}(\theta)$ with $K_{s}^{\prime}(\theta)$. In rapidity space, the latter is given by a discrete family of Cauchy distributions

$$
K_{s}(\theta)=\frac{1}{2 \pi \mathrm{i}} \partial_{\theta} \log S_{s}(\theta)=\frac{1}{2 \pi} \frac{s}{\theta^{2}+(s / 2)^{2}} .
$$

Notice that for any finite fixed rapidity $\theta$, the density of states $\rho_{a, s}^{\text {tot }}$ decays as about $1 / s^{2}$ in the large-s limit. However, when integrated against any dummy integrable function $f(\theta)$ as in GHD, we have the following large- $s$ properties:

$$
\begin{aligned}
\int_{\mathbb{R}} d \theta f(\theta) \rho_{a, s}^{\mathrm{tot}}(\theta) & \sim \frac{1}{s}, \\
\int_{\mathbb{R}} d \theta f(\theta) v_{a, s}^{\mathrm{eff}}(\theta) & \sim \frac{1}{s},
\end{aligned}
$$

for every quasiparticle flavor $a$.

As explained in the main text, these properties allow us to infer the superdiffusive scaling of charge dynamics with an algebraic dynamical exponent $z=3 / 2$.

Nonfundamental on-site representations. The large-s scaling properties (B14) and (B15) likewise hold in models with nonfundamental local degrees of freedom, namely, for any on-site finite-dimensional unitary irreducible representations $\mathcal{V}_{\Lambda}$. At the level of algebraic dressing equations, this amounts to placing the source terms in a different (generic) position.

As a quick illustration, we derive the explicit solution to the $\mathrm{SU}(3)$ chain in the adjoint representation $(\mathbf{8})$, with Dynkin labels $\Lambda_{\mathrm{p}}=\omega_{1}+\omega_{2} \equiv[1,1]$. By virtue of $\mathbb{Z}_{2}$ invariance of the dressing equations (under interchanging the flavors), we have $F_{1, s}=F_{2, s}$, and therefore

$$
s^{-1} \star F_{a, s}-I_{s, s^{\prime}}^{A_{\infty}} \bar{n}_{s^{\prime}} F_{a, s^{\prime}}-n_{s} F_{a, s}=\delta_{s, 1},
$$

where $I_{s, s^{\prime}}^{A_{\infty}}=\delta_{s, s^{\prime}-1}+\delta_{s, s^{\prime}+1}$. The solution reads

$$
\begin{aligned}
F_{a, s}= & \frac{1}{3}\left[\frac{s+2}{s} K_{s}-\frac{s+1}{s+3} K_{s+3}\right] \\
& +\frac{1}{3}\left[K_{s+1}-K_{s+2}\right] .
\end{aligned}
$$

\section{APPENDIX C: INTEGRABLE QUANTUM FIELD THEORIES}

In the main text, we confined ourselves exclusively to integrable ferromagnetic quantum chains. Nonetheless, these do not exhaust the list of integrable quantum models invariant under global symmetries of a non-Abelian Lie group. As briefly mentioned in the conclusions, there are other types of quantum chains, such as most prominently integrable fermionic models that exhibit Lie supersymmetries $\left(\mathbb{Z}_{2}\right.$-graded Lie algebras), which are on the same 
grounds expected to reveal charge superdiffusion with the exponent $z=3 / 2$; see, e.g., Refs. [38,46] for concrete examples.

More remarkably, however, anomalous charge transport likewise takes place in integrable quantum field theories in two space-time dimensions, provided that elementary particles carry internal isotropic degrees of freedom, which, in the classical action, take values either in Lie groups $G$ or coset manifolds $G / H$. The two most prominent classes of integrable quantum field theories (IQFT) of this kind are the $\mathrm{O}(n)$ nonlinear sigma models (NLSM) and the principal chiral fields (PCF) on $G \times G$; cf. Refs. [150-155] for more details.

Even though Goldstone modes of Lorentz-invariant systems always comprise linearly dispersing (i.e., type-I) modes, one should keep in mind that we are interested here exclusively in the time evolution of the Noether currents and not correlation functions amongst individual components of quantum fields. A crucial observation in this respect is that the temporal components of the Noether twocurrents may be understood as (quantized) spin waves. Moreover, in finite-temperature ensembles, excitations of these internal interacting degrees of freedom undergo nontrivial dressing. The situation in fact mirrors that of the Heisenberg spin chains or their higher-rank analogues we investigated earlier. We do not attempt to give a comprehensive exposition but rather demonstrate the basic principles on the $\mathrm{O}(3)$ NLSM, representing a paradigmatic, integrable, interacting QFT with nondiagonal scattering. We briefly outline the differences in a few other IQFTs that display $\mathrm{SU}(2)$ symmetry while postponing a detailed quantitative study of other integrable nonlinear sigma models on Riemannian symmetric spaces $G / H$ [156] and their classical limits for another study.

Another widely studied class of integrable system that exhibit global non-Abelian symmetries are nonrelativistic QFTs commonly known as the Yang-Gaudin models [157-161]. They describe one-dimensional interacting Bose or Fermi gases with internal degrees of freedom (see, e.g., Refs. [162,163] for recent applications of GHD).

\section{1. $O(3)$ nonlinear sigma model}

To begin, we spell out some of the main properties of the $\mathrm{O}(3)$ NLSM quantum field theory, employing the Hamiltonian formalism. The quantum field $\hat{\mathbf{n}}=\left(\hat{n}^{x}, \hat{n}^{y}, \hat{n}^{z}\right)$, subjected to the nonlinear constraint $\hat{\mathbf{n}} \cdot \hat{\mathbf{n}}=1$, transforms in the vector representation of $\mathrm{O}(3)$. Since the vacuum stability subgroup with respect to the polarization axis (say, $\hat{n}^{z}=1$ ) is $\mathrm{O}(2)$, the target manifold for $\mathrm{O}(3) \mathrm{NLSM}$ is $\mathrm{O}(3) / \mathrm{O}(2) \cong S^{2}$. Introducing the momentum $\hat{\boldsymbol{\pi}}$ conjugate to $\hat{\mathbf{n}}$ and the angular-momentum field $\hat{\mathbf{m}}=\hat{\mathbf{n}} \times \hat{\boldsymbol{\pi}}$ perpendicular to $\hat{\mathbf{n}}, \hat{\mathbf{m}} \cdot \hat{\mathbf{n}}=0$, the Hamiltonian density (here, without the topological $\Theta$ term) has the form

$$
\left.H_{\mathrm{O}(3)}=\frac{v}{2} \int d x\left[g \hat{\mathbf{m}}^{2}+g^{-1} \hat{\mathbf{n}}_{x}^{2}\right)\right]
$$

The conserved Noether two-current associated with global $\mathrm{O}(3)$ rotations is given by

$$
\partial_{\mu} \hat{\mathbf{j}}_{\mu}=0, \quad \hat{\mathbf{j}}_{\mu}=g^{-1} \hat{\mathbf{n}} \times \partial_{\mu} \hat{\mathbf{n}},
$$

for $\mu \in\{x, t\}$. The integrated magnetization density provides a local conservation law, $(d / d t) \int d x \hat{\mathbf{m}}(x)=0$.

The O(3) NLSM arises as the effective low-energy theory of (Haldane) spin- $S$ antiferromagnets, where one identifies $v=2 J S$, while the coupling strength is given by $g=2 / S$, assuming large $S$; the $S^{2}$ field $\hat{\mathbf{n}}$ governs fluctuations of the staggered order parameter above the antiferromagnetic (Néel) ground state, whereas $\hat{\mathbf{m}}$ pertains to ferromagnetic (spin-wave) fluctuations. Elementary excitations of Eq. (C1) are an $\mathrm{SU}(2)$ triplet of massive spinful bosons with relativistic (bare) dispersion $e(p)=\sqrt{p^{2}+\Delta^{2}}$. As is customary, relativistic dispersion of massive particles can be parametrized by a rapidity variable $\theta$,

$$
e(\theta)=\Delta \cosh (\theta), \quad p(\theta)=\Delta \sinh (\theta) .
$$

The mass $\Delta$ gets generated dynamically via dimensional transmutation and vanishes in the large- $S$ limit. At weak coupling $(g \rightarrow 0)$, the theory becomes effectively free with $\operatorname{dim}(G / H)=2$ massless (Goldstone) bosons.

Because of integrability, the model possesses infinitely many "hidden" conserved currents and a completely factorizable many-body scattering matrix. Notice, however, that exchange of isotropic degrees of freedom upon elastic interparticle interactions renders the scattering matrix nondiagonal, signifying that particles, upon collision, do not only pick up a phase factor but can also change their state (internal quantum numbers). This feature has a direct analogy with the nested spin chains.

The O(3) NLSM can be diagonalized by means of the algebraic Bethe ansatz, initially carried out in Ref. [59] (see also Refs. [156,164] for a more comprehensive discussion). The standard trick to resolve the exchange of isotropic degrees of freedom is to introduce additional (called auxiliary) spin-wave excitations (magnons) - the extended basis of excitations that renders the scattering diagonal. These auxiliary magnons are, despite being massless, regarded as independent quasiparticles. Imposing the finite-volume boundary condition, one arrives at the following nested Bethe Ansatz equations [59]:

$$
\begin{gathered}
e^{\mathrm{i} p\left(\theta_{j}\right) L} \prod_{k=1}^{N_{\theta}} S\left(\theta_{j}, \theta_{k}\right) \prod_{l=1}^{N_{\lambda}} S^{-1}\left(\theta_{j}, \lambda_{l}\right)=-1, \\
\prod_{k=1}^{N_{\theta}} S^{-1}\left(\lambda_{j}, \theta_{k}\right) \prod_{l=1}^{N_{\lambda}} S\left(\lambda_{j}, \lambda_{l}\right)=-1,
\end{gathered}
$$


belonging to the sector with $N_{\theta}$ momentum-carrying physical excitations and $N_{\lambda}$ magnons (with associated rapidity $\lambda$ ). There is a single rational scattering amplitude reading $S(\theta)=(\theta-\mathrm{i} \pi / 2) /(\theta+\mathrm{i} \pi / 2)$. Looking at Eq. (C5), it is evident that interactions among the magnons are identical to those in the isotropic Heisenberg SU(2) chain, resulting in the formation of bound states (Bethe strings).

Taking the thermodynamic limit, obtained by sending $L \rightarrow \infty$ with ratios $N_{\theta} / L$ and $N_{\lambda} / L$ finite, and introducing the physical density $\rho_{0}(\theta)$ and auxiliary densities of the Bethe $s$-strings $\rho_{s \geq 1}(\theta)$, one arrives at the following (decoupled) Bethe-Yang equations

$$
\begin{gathered}
\rho_{0}^{\mathrm{tot}}=\frac{p^{\prime}}{2 \pi}+s \star \bar{\rho}_{2}, \\
\rho_{s}^{\mathrm{tot}}=\delta_{s, 2} s \star \rho_{0}+s \star I_{s, s^{\prime}}^{A_{\rho^{\infty}}} \bar{\rho}_{s^{\prime}} .
\end{gathered}
$$

By performing an extra particle transformation on the physical node, $\mathcal{Y}_{0} \mapsto 1 / \mathcal{Y}_{0}$ (i.e., identifying $\mathcal{Y}_{0} \equiv \rho_{0} / \bar{\rho}_{0}$ ), the TBA equations can, once again, be brought into the canonical $\mathcal{Y}$-system format

$$
\log \mathcal{Y}_{s}=-\delta_{s, 0} \beta e+s \star I_{s, s^{\prime}}^{D_{\infty}} \log \left(1+\mathcal{Y}_{s^{\prime}}\right),
$$

with the incidence matrix of the $D_{\infty}$ Dynkin diagram (see Fig. 7). It is worth noticing that the U(1) chemical potential $h$ enters only implicitly via the asymptotics of the $\mathcal{Y}$ functions, $\lim _{s \rightarrow \infty} \log (1 / s) \log \mathcal{Y}_{s}=h$. The $\mathrm{O}(3)$ model is, in fact, a member of an infinite family of integrable sigma models with $\mathrm{SU}(n) / \mathrm{SO}(n)$ target spaces, with $n-1$ massive nodes whose thermodynamic particle content gets arranged according to $A_{n-1} \times D_{\infty}$ Dynkin diagrams [156].

Since QFT are not UV complete, there is no regime in which the TBA equations would be rendered algebraic. Despite the fact that there is no issue with computing the thermodynamic $\mathcal{Y}$ functions in the $\beta \rightarrow 0$ limit, say, at half filling $h=0$, the equations for the densities, Eqs. (C7), yield UV-divergent rapidity integrals. This type of divergence is often cured by hand by imposing a momentum cutoff, such as, e.g., in Ref. [165]. We purposely avoid it here since this would invariably break integrability and thereby spoil the late-time decay of the charge correlations. To ensure convergence of rapidity integrals, temperature $\beta>0$ has to be taken into account in a nonperturbative way. The only safe way to introduce a UV cutoff while preserving integrability is via a full light-cone discretization. This discretization has been achieved for the case of SU(2) PCF in Ref. [153], which demonstrates how the integrable QFT arises from a particular continuum scaling limit of a staggered quantum spin chain.

In the scope of physics applications, one is typically interested in the opposite regime of low temperatures. The low-temperature expansion of Eqs. (C7) and (C8) can be found in Ref. [109]. We do not reproduce the results here but merely reserve a few remarks. In order to properly
$\mathrm{SU}(2) \mathrm{cGN}$

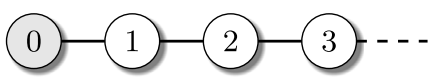

O(3) NLSM

$\Theta=0$

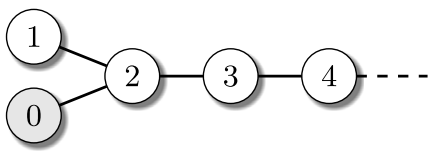

$\mathrm{O}(3)$ NLSM

$\Theta=\pi$
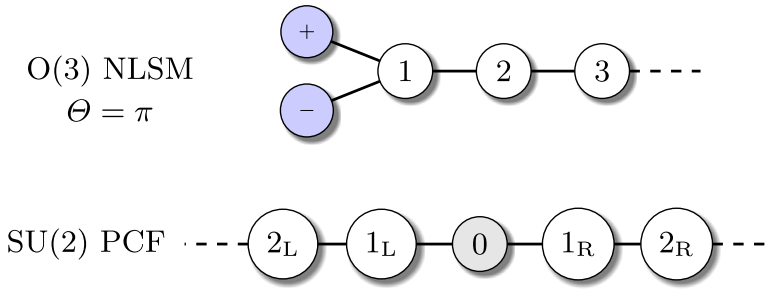

FIG. 7. TBA incidence diagrams depicting the complete thermodynamic quasiparticle spectra for a number of paradigmatic examples of massive and massless IQFTs with isometry groups of rank one: $\mathrm{SU}(2)$ chiral Gross-Neveu model, $\mathrm{O}(3)$ nonlinear sigma model, with and without the topological $\Theta$ term and $\mathrm{SU}(2)$ principal chiral field, appearing from top to bottom in the respective order. Massive physical excitations, with bare dispersion [Eq. (C3)], are designated by gray nodes. Massless physical excitations, with bare dispersion [Eq. (C9)], are marked in blue. The structure of TBA equations is a direct product of two types of Dynkin diagrams: a finite one associated with the isometry group of the elementary physical excitations (marked with color nodes), and an infinite one of type $A$ associated with a tower of auxiliary magnons (white nodes), whose bijectives correspond to finite-dimensional irreducible $\mathfrak{s} \mathfrak{t}(2)$ representations.

account for the effects of thermal fluctuations in a finitedensity state, even at arbitrarily low temperatures $T>0$, it is crucial to properly account for all the contributions of the spin-wave excitations. Magnonic excitations turn out to be inconsequential only in the regime $h / T \gg 1$, where the theory can be well approximated by the semiclassical description developed in Refs. [166-168]. In stark contrast, when approaching the unmagnetized (half-filled) state (i.e., for $h / T \ll 1)$, the contributions to the spin diffusion due to giant magnons become amplified, and, in analogy to the isotropic Heisenberg chain, one accordingly finds a diverging spin diffusion constant $D_{s} \sim 1 / h$ accompanied by a vanishing-spin Drude weight.

Topological term. It is well known that the O(3) NLSM admits an integrable deformation with the inclusion of the topological $\Theta$ term with angle $\Theta=\pi[59,169]$ [describing the low-energy limit of $\mathrm{SU}(2)$-invariant spin chains with odd spin $S$; in general, $\Theta=2 \pi S$ [170] ]. This term has a profound effect on the low-energy spectrum; instead of a massive triplet, one instead finds an $\mathrm{SU}(2)$ doublet of massless elementary excitations, with bare dispersion

$$
e_{ \pm}(\theta)= \pm p(\theta)=\frac{M}{2} e^{ \pm \theta}
$$


where \pm designate the right $(p>0)$ and left $(p<0)$ moving fields. The theory is massless but not scale invariant; $M$ provides a mass scale that only affects the left-right scattering processes. At the level of the TBA description, the physical species are comprised of the left and right movers with densities $\rho_{ \pm}(\theta)$ [59]. The internal magnon structure, however, remains intact [59]. For instance, the TBA equations in the quasilocal form are now of the form

$$
\begin{aligned}
\log \mathcal{Y}_{ \pm}= & \beta \frac{M}{2} e^{ \pm \theta}+s \star \log \left(1+\mathcal{Y}_{1}\right) \\
\log \mathcal{Y}_{s}= & \delta_{s, 1} s \star\left(1+\mathcal{Y}_{+}\right)\left(1+\mathcal{Y}_{-}\right) \\
& +s \star I_{s, s^{\prime}}^{A_{\infty}} \log \left(1+\mathcal{Y}_{s^{\prime}}\right)
\end{aligned}
$$

Further details can be found in Ref. [109] and references therein.

Classical picture. We stress that magnons in QFTs are not due to quantization but are rather a consequence of the rotational symmetry. Thus, magnons are already featured at the classical level. The $\mathrm{O}(3)$ nonlinear sigma model represents an integrable classical field theory [171]. The Euler-Lagrange equation for the classical field $\mathbf{n} \in S^{2}$ reads (in dimensionless units) [172]

$$
\mathbf{n}_{t t}-\mathbf{n}_{x x}+\left(\mathbf{n}_{t}^{2}-\mathbf{n}_{x}^{2}\right) \mathbf{n}=0 .
$$

Owing to Lorentz invariance, linear fluctuations above a degenerate ground state are comprised of two, transversal, linearly dispersing (type-I) Goldstone modes. However, we are interested in the time evolution of the temporal component of the conserved Noether two-current, which presently corresponds to the ferromagnetic order parameter $\mathbf{m}=\mathbf{n} \times \boldsymbol{\pi}$. Its equation of motion simply reads $\mathbf{m}_{t}=\mathbf{n} \times \boldsymbol{\pi}_{t}$. In terms of the Hamiltonian equations,

$$
\mathbf{n}_{t}=\boldsymbol{\pi}, \quad \boldsymbol{\pi}_{t}=\mathbf{n}_{x x}+\left(\mathbf{n}_{x}^{2}-\boldsymbol{\pi}^{2}\right) \mathbf{n},
$$

one can readily deduce the equation of motion for the angular-momentum field,

$$
\mathbf{m}_{t}=\mathbf{n} \times \mathbf{n}_{x x}, \quad \mathbf{m}^{2}=\boldsymbol{\pi}^{2} .
$$

This result reveals the mechanism for how spatial fluctuations of field $\mathbf{n}(x, t)$ induce the dynamics of magnetization $\mathbf{m}(x, t)$. In the quantum version of the O(3) NLSM, fluctuations of these magnetization waves carrying integer quanta scatter completely elastically off one another, as described by Eqs. (C5).

\section{Other integrable QFTs}

There are many other integrable QFTs with non-Abelian isometry groups that can be treated along the lines of the O(3) NLSM; see, e.g., Refs. [155,173]. The best-studied examples are $\mathrm{O}(n)$ NLSMs on a hypersphere $S^{n-1} \cong$ $\mathrm{O}(n) / \mathrm{O}(n-1)$, with the Lagrangians

$$
\mathcal{L}_{\mathrm{O}(n)}=\frac{1}{2 g} \int d x\left(\partial_{\mu} \hat{\mathbf{n}}\right)^{2}, \quad \hat{\mathbf{n}}^{2}=1 .
$$

In the simply laced cases, that is, $\mathrm{O}(2 r)$ with $r \geq 4$ [174], the thermodynamic spectrum of excitations comprises $r$ flavors of quasiparticles (one per node in the $D_{r}$ Dynkin diagram), each of which further binds into bound states (the ordinary $s$ strings). The O(4) NSLM in the fundamental (vector) representation is special as it can be represented as the $\mathrm{SU}(2)_{\mathrm{L}} \times \mathrm{SU}(2)_{\mathrm{R}}$ principal chiral field with particles transforming in the bifundamental representation of $\mathfrak{g} \mathfrak{t}(2)$ [175]. Its spectrum involves massive spinful particles with two types of SU(2) spins - these are elementary excitations above the Fermi sea (antiferromagnetic ground state) in an $\mathrm{SU}(2)$ spin- $S$ chain. Its thermodynamic particle content is graphically represented in Fig. 7. In this respect, one can view (using a loose analogy) the SU(2) PCF as the bosonic QFT counterpart of the Fermi-Hubbard model [72,73]. In the strong-coupling limit, the SU(2) PCF model splits up into two copies of the isotropic Heisenberg chain. The classical limit and the associated Riemann-Hilbert equations can, once again, be derived from the asymptotic Bethe equations [173], governing the regime with $N \rightarrow \infty$ particles with large rapidities $\theta \sim \mathcal{O}(N)$ [with the quantity $\Delta L=\exp (-2 \pi N)$ playing the role of a small parameter].

Another prominent class of integrable QFTs are the $\mathrm{SU}(n)$ chiral Gross-Neveu models (cGN) [176]. We take a brief look at the simplest SU(2) case, which describes two interacting Dirac fermions expressed in terms of twocomponent spinors $\hat{\psi}_{a}(a=1,2)$ with Lagrangian density

$$
\begin{aligned}
\mathcal{L}_{\mathrm{cGN}}= & \mathrm{i} \hat{\bar{\psi}}_{a} \not \partial \hat{\psi}^{a}+\frac{1}{2} g_{s}^{2}\left(\left(\hat{\bar{\psi}}_{a} \hat{\psi}^{a}\right)^{2}-\left(\hat{\bar{\psi}}_{a} \gamma_{5} \hat{\psi}^{a}\right)^{2}\right) \\
& -\frac{1}{2} g_{v}^{2}\left(\hat{\bar{\psi}}_{a} \gamma_{\mu} \hat{\psi}^{a}\right)^{2},
\end{aligned}
$$

with $\not \partial \equiv \gamma_{\mu} \partial^{\mu}$ and $\gamma$ matrices $\gamma_{0}=\sigma_{1}, \gamma_{1}=\mathrm{i} \sigma_{2}, \gamma_{5}=\gamma_{0} \gamma_{1}$ obeying the Clifford algebra $\gamma_{\mu}, \gamma_{\nu}=2 \eta^{\mu \nu}$ with metric tensor $\eta=\operatorname{diag}(1,-1)$. Lagrangian (C16) is symmetric under $\mathrm{U}(2) \times \mathrm{U}(1)_{c}$; spinors transform in the fundamental representation of $\mathrm{U}(2)$, whereas $\mathrm{U}(1)_{c}$ is associated with the chiral symmetry $\hat{\psi} \rightarrow e^{\mathrm{i} \theta \gamma_{5}} \hat{\psi}$. The spectrum of the model involves a single $\mathrm{SU}(2)$ multiplet of massive fermions, with relativistic dispersion $e(\theta)=\Delta \cosh (\pi \theta / 2)$ and $p(\theta)=$ $\Delta \sinh (\pi \theta / 2)$ [aside from the massless excitation charged under $\mathrm{U}(1)_{c}$ that completely decouples]. The amplitude of a two-fermion scattering is given by $S(\theta)=-\Gamma[1-$ $(\theta / 4 i)] \Gamma\left[\frac{1}{2}+(\theta / 4 i)\right] / \Gamma[1+(\theta / 4 i)] \Gamma\left[\frac{1}{2}-(\theta / 4 i)\right]$; see, e.g., Refs. [76,177]. It is of primary importance that the scattering of fermions carrying opposite spin projections is, once again, identical to the spin exchange of the $\mathrm{SU}(2)$ Heisenberg chain. To exhibit this similarity, we perform the particle-hole transformation on the zeroth node (assigned 
to physical, i.e., fermionic, density), $\mathcal{Y}_{0} \mapsto 1 / \mathcal{Y}_{0}$, yielding the Dynkin-type TBA equations with the incidence matrix of the $A_{1} \times A_{\infty}$ diagram [177],

$\log \mathcal{Y}_{s}=-\delta_{s, 0} \beta e+s \star \log \left(1+\mathcal{Y}_{s-1}\right)\left(1+\mathcal{Y}_{s+1}\right)$.

In this labeling, the $s$ strings sit at nodes $s \geq 1$. Apart from an extra massive particle assigned to the initial node $s=0$, the obtained equations are indeed those of the isotropic Heisenberg spin-1/2 chain (see Fig. 7). A family of integrable Gross-Neveu models with $\mathrm{SU}(n)$ symmetry with $n-1$ coupled copies of Eqs. (C17), resulting in the TBA incidence matrices of the type $A_{n-1} \times A_{\infty}$. There are also the Gross-Neveu models with $\mathrm{O}(2 n)$ symmetry, which have been described in Ref. [156].

Numerical analysis. In order to extract the large-s asymptotic properties of thermodynamic state functions, we have numerically solved the TBA equations for the above SU(2)-invariant IQFTs with a common magnon structure. The deduced scaling properties match those of their spin-chain counterparts, as specified by Eqs. (B14) and (B15), indicating, once again, superdiffusive transport with the dynamical exponent $z=3 / 2$.

\section{Multicomponent Fermi gases}

For completeness, we finally give another representative example of an integrable QFT. We consider the YangGaudin field theory of spinful fermions-the fermionic cousin of the integrable two-component Bose gas. Our purpose here is mainly to achieve unification with other integrable systems characterized by the anomalous dynamical exponents $z=3 / 2$. There are two conserved Noether charges involved, representing the conserved electron charge and total magnetization. As we clarify below, anomalous transport occurs only in the spin sector, provided the nonAbelian symmetry is not explicitly broken by the finite chemical potential. On the contrary, transport of electron charge [Noether charge associated to a $\mathfrak{t}(1)$ subalgebra] behaves ordinarily; i.e., it is characterized by a finite-charge Drude weight with a regular diffusive correction.

To elucidate the underlying algebraic structure, we note that integrable multicomponent Fermi (and Bose) gases arise as continuum limits of integrable lattice models with $\mathcal{U}_{q}(\mathfrak{g l}(n))$ symmetry (commonly known in the integrability literature as the Perk-Schultz models) in the undeformed $q \rightarrow 1$ limit [upon which the global $S U(2)$ invariance gets restored]. Which field theory one ends up with depends, besides the rank $r=n-1$ of $\mathfrak{g}$, also on the choice of $\mathbb{Z}_{2}$ grading (without the grading, one can similarly produce multicomponent integrable Bose gases). The specific instance of a two-component Fermi gas that we are revisiting here arises as a continuum limit of the rank-2 Perk-Schultz model with $\mathcal{U}_{q}(\mathfrak{g l}(2 \mid 1))$ symmetry in $(-,+,+)$ grading $[161,178,179]$. As we clarify below, the resulting TBA equations algebraically match those of an integrable fermionic chain with the global symmetry of $S U(2 \mid 1)$ Lie superalgebra, representing the $t-J$ model of spin- $1 / 2$ fermions at the supersymmetric point $J=2 t$,

$$
\begin{aligned}
H_{\mathrm{SU}(2 \mid 1)}= & P\left[-t \sum_{j=1}^{L} \sum_{\sigma \in \uparrow, \downarrow}\left(c_{j, \sigma}^{\dagger} c_{j+1, \sigma}+c_{j+1, \sigma}^{\dagger} c_{j, \sigma}\right)\right] P \\
& +J \sum_{j=1}^{L}\left(S_{j} \cdot S_{j+1}-\frac{1}{4} n_{j} n_{j+1}\right)
\end{aligned}
$$

where $P=\prod_{j=1}^{L}\left(1-n_{j, \uparrow} n_{j, \downarrow}\right)$ projects out the doubly occupied states.

Two-component Yang-Gaudin Fermi gas. The Hamiltonian density of the Yang-Gaudin model with coupling constant $c>0$ reads (in units $m=\hbar=1$ )

$\mathcal{H}_{\mathrm{YG}}=\frac{1}{2} \hat{\Psi}_{x}^{\dagger} \hat{\Psi}_{x}+\frac{c}{2}:\left(\hat{\Psi}^{\dagger} \hat{\Psi}\right)^{2}:-\tilde{\mu} \hat{\Psi}^{\dagger} \Psi-\tilde{h} \hat{\Psi}^{\dagger} \sigma^{z} \hat{\Psi}$

expressed in terms of a two-component spinor field $\hat{\Psi}(x)=\left(\hat{\psi}_{\uparrow}(x), \hat{\psi}_{\downarrow}(x)\right)^{\mathrm{T}}$, with external fields $\tilde{\mu}$ and $\tilde{h}$ coupling to charge and spin degrees of freedom, respectively. At the level of grand-canonical Gibbs ensembles, we introduced the corresponding chemical potentials $\mu \equiv \beta \tilde{\mu}$ and $h \equiv \beta \tilde{h}$. Notice that, sometimes, the model includes an additional (integrability-breaking) potential term $V(x) \hat{\Psi}^{\dagger} \hat{\Psi}$, which we have to exclude.

The model is exactly solvable by the nested Bethe ansatz. Consider an eigenstate of $N$ spin-1/2 electrons in a periodic system of length $L$, of which $N_{\uparrow}$ and $N_{\downarrow}$ correspond to the number of electrons with up and down $z$ components. The electron bare dispersion is not nonrelativistic, and it assumes a quadratic dependence on the momentum $\theta$, written $e_{0}(\theta)=\theta^{2}$. The variable $\theta$ can thus be regarded as the rapidity, and subsequently, we relabel it as $\theta \rightarrow \theta^{(1)}$, with the superscript label referring to the physical (i.e., momentum-carrying) excitations. The total energy of an eigenstate in the presence of fields reads $E=\sum_{i=1}^{N} e_{0}\left(\theta_{i}^{(1)}\right)-\tilde{\mu} N-\tilde{h}\left(N_{\uparrow}-N_{\downarrow}\right)$. In analogy to the $\mathrm{O}(3)$ NLSM and other systems with nested spectra, a dynamical lattice of propagating electrons supports interacting spin waves. The latter are associated with auxiliary rapidities $\theta_{j}^{(2)}$. For a periodic system of size $L$ with $N \equiv$ $N_{\uparrow}+N_{\downarrow}$ electrons, the two sets of rapidities are determined as solutions to the following nested Bethe equations:

$$
\begin{gathered}
e^{\mathrm{i} \theta_{i}^{(1)} L} \prod_{j=1}^{N_{\downarrow}} S_{1}\left(\theta_{i}^{(1)}, \theta_{j}^{(2)}\right)=1, \\
\prod_{j \neq i}^{N_{\downarrow}} S_{1,1}\left(\theta_{i}^{(2)}, \theta_{j}^{(2)}\right) \prod_{j=1}^{N} S_{1,1}^{-1}\left(\theta_{i}^{(2)}, \theta_{j}^{(1)}\right)=1,
\end{gathered}
$$


where in the first (second) equation, the index $i$ runs from 1 to $N\left(N_{\downarrow}\right)$, and $S_{j}(\theta)=(\theta-j \mathrm{ic} / 2) /(\theta+j \mathrm{ic} / 2), S_{1,1}(\theta) \equiv$ $S_{2}(\theta)$ are the standard elementary scattering amplitudes, with the associated scattering kernels

$$
K_{j}(\theta)=\frac{1}{2 \pi \mathrm{i}} \partial_{\theta} \log S_{j}(\theta)=\frac{1}{2 \pi} \frac{c j}{\theta^{2}+(j c / 2)^{2}},
$$

or equivalently, in Fourier space, $K_{j}(k)=e^{-j|k| c / 2}$.

Passing to the thermodynamic limit, and introducing rapidity densities of unbound electrons $\rho_{0}(\theta)$ and the standard Bethe $s$ strings $\rho_{s}(\theta)$, the Bethe-Yang equations take the following quasilocal form:

$$
\begin{gathered}
\rho_{0}^{\mathrm{tot}}=\frac{1}{2 \pi}+\left(K_{1} \star s\right) \star \rho_{0}-s \star \bar{\rho}_{1}, \\
\rho_{1}^{\mathrm{tot}}=s \star\left(\rho_{0}+\bar{\rho}_{2}\right), \\
\rho_{s \geq 2}^{\mathrm{tot}}=s \star\left(\bar{\rho}_{s-1}+\bar{\rho}_{s+1}\right),
\end{gathered}
$$

with the $s$ kernel $s(\theta)=(2 c \cosh (\pi \theta / c))^{-1}$, satisfying $1 \star s=1 / 2$ and $s(k)=\left(e^{c|k| / 2}\right) /\left(1+e^{c|k|}\right)$. Notice that the source term comes from $(1 / 2 \pi) \partial_{\theta} k_{0}(\theta)=1 / 2 \pi$. The energy density of an equilibrium macrostate is then given by

$e=\int \mathrm{d} \theta\left(e_{0}(\theta)-\tilde{\mu}\right) \rho_{0}(\theta)+\sum_{s \geq 1} 2 s \tilde{h} \int \mathrm{d} \theta \rho_{s}(\theta)$,

whereas electron charge and magnetization density read $n_{e}=1 \star \rho_{0}$ and $m=\frac{1}{2}\left(1 \star \rho_{0}\right)-\sum_{s \geq 1} s\left(1 \star \rho_{s}\right)$. Unlike the density of magnetization, electron density is a strictly positive quantity in any equilibrium macrostate, which readily implies that the Drude weight of the electron charge always stays strictly positive.

The TBA equations in terms of the thermodynamic $\mathcal{Y}$ functions $\mathcal{Y}_{A}(\theta)=\bar{\rho}_{A}(\theta) / \rho_{A}(\theta)$, on the other hand, read

$$
\begin{aligned}
\log \mathcal{Y}_{0}= & \beta \theta^{2}-\mu-\left(K_{1} \star s\right) \star \log \left(1+1 / \mathcal{Y}_{0}\right) \\
& -s \star \log \left(1+\mathcal{Y}_{1}\right),
\end{aligned}
$$

$\log \mathcal{Y}_{1}=-s \star \log \left(1+1 / \mathcal{Y}_{0}\right)+s \star \log \left(1+\mathcal{Y}_{2}\right)$,

$$
\log \mathcal{Y}_{s \geq 2}=s \star I_{s, s^{\prime}}^{A_{\infty}} \log \left(1+\mathcal{Y}_{s^{\prime}}\right),
$$

with $I_{s, s^{\prime}}^{A_{\infty}}=\delta_{s, s^{\prime}+1}+\delta_{s, s^{\prime}-1}$. Importantly, the chemical potential $h$ coupling to total magnetization enters, as usual, through the large-s asymptotics

$$
\lim _{s \rightarrow \infty} \frac{\log \mathcal{Y}_{s}}{s}=2 h
$$

Finally, the equilibrium free energy density in the grandcanonical Gibbs states reads

$$
-\beta f=\frac{1}{2 \pi} \int d \theta \log \left(1+1 / \mathcal{Y}_{0}(\theta)\right) .
$$

Let us mention, in passing, that the above TBA equations coincide, apart from the dispersion-dependent source terms, with those of the fundamental $S U(2 \mid 1)$ quantum chain [the supersymmetric $t-J$ model given by Eq. (C18)] in the distinguished grading $\odot-\otimes$. For further details, we refer the reader to Ref. [46], where the complete solution to the algebraic TBA equations for the $S U(2 \mid 1)$ chain is derived.

\section{a. Dressing equations}

In the scope of our study, we are primarily interested in the dressed dispersion relation and the dressed Noether charges. There are three different limits of the above BetheYang and TBA equations that are analytically tractable, all treated originally in Ref. [160]: (i) the zero-temperature limit $T \rightarrow 0$, (ii) the weak-coupling limit $c \rightarrow 0$, and (iii) the strong-coupling limit $c \rightarrow \infty$. Since none of these limits can be regarded as generic local equilibrium states, they are not really relevant to us. On the other hand, there is not UV completeness in the high-temperature limit, as in relativistic integrable QFTs. Even though the $\mathcal{Y}$ functions become flat (i.e., independent of rapidity $\theta$ ) in the $\beta \rightarrow 0$ limit, there is no regulator at large rapidities to ensure convergence of the free energy density.

It is nevertheless instructive to exhibit a closed-form solution to the TBA equation in the limit $\beta \rightarrow 0$ while keeping chemical potentials $\mu$ and $h$ general. Denoting the constant thermodynamic $\mathcal{Y}$ functions by $\mathcal{Y}_{s \geq 0}$, and using $1 \star s=1 / 2$ and likewise $\left(K_{1} \star s\right) \star 1=1 / 2$, the TBA equations are readily rendered algebraic:

$$
\begin{aligned}
e^{2 \mu} \mathcal{Y}_{0}^{2} & =1 /\left[\left(1+1 / \mathcal{Y}_{0}\right)\left(1+\mathcal{Y}_{1}\right)\right], \\
\mathcal{Y}_{1}^{2} & =\left(1+\mathcal{Y}_{2}\right) /\left(1+1 / \mathcal{Y}_{0}\right), \\
\mathcal{Y}_{s \geq 2}^{2} & =\left(1+\mathcal{Y}_{s-1}\right)\left(1+\mathcal{Y}_{s+1}\right) .
\end{aligned}
$$

Notice, once more, that the charge chemical potential $\mu$ remains explicitly present while $h$ implicitly hides in the asymptotic condition (C30). The solution to the three-term recursion for $\mathcal{Y}_{s \geq 1}$ in the bulk that satisfies the prescribed asymptotics at large $s$ is given by

$$
\mathcal{Y}_{s \geq 1}=y_{s}^{2}-1, \quad \mathcal{Y}_{0}=\frac{y_{0}^{2}}{1-y_{0}^{2}}
$$

We have introduced the functions 


$$
y_{s}(a, b)=\frac{b a^{s}-b^{-1} a^{-s}}{a-a^{-1}},
$$

which obey the Hirota bilinear relation, $y_{s}^{2}-y_{s-1} y_{s+1}-$ $1=0$. The functions $y_{s}$ are none other than classical characters associated to a non-semi-simple Lie algebra $\mathfrak{g} \mathfrak{t}(2) \oplus \mathfrak{t}(1)$, which is the bosonic subalgebra of the Lie supergroup $S U(2 \mid 1)$, specifically, $y_{s}$ specialized to $\mathfrak{g} \mathfrak{u}(2)$ characters if $b=a$. The two remaining free constants $a$ and $b$, which are functions of $h$ and $\mu$, can be determined from the boundary condition $\mathcal{Y}_{0}\left(1+\mathcal{Y}_{0}\right)\left(1+\mathcal{Y}_{1}\right)=e^{-2 \mu}$. The large- $s$ asymptotics is satisfied by $a=e^{-h}$. The coefficient $b$ is then obtained from

$$
\frac{a^{2}\left(b^{2}-1\right)^{2}}{\left(a^{2}-b^{2}\right)^{2}}=c^{2}, \quad c \equiv e^{-\mu},
$$

yielding

$$
b(\mu, h)=\sqrt{\frac{1+c a}{1+c / a}}, \quad \mathcal{Y}_{0}=\frac{a c^{2}}{a+c+a^{2} c} .
$$

Reparametrizing the $y$ characters in terms of chemical potentials $\mu$ and $h$, we have the form $y_{s}(\mu, h)=$ $\sinh ^{2}(s h+\mu) / \sinh ^{2}(h)$.

In the $\beta \rightarrow 0$ limit, the Fermi occupation functions, $n_{s \geq 0}(\mu, h)=1 /\left(1+\mathcal{Y}_{s}(\mu, h)\right)$, can be expressed in the form

$n_{0}(\mu, h)=1-y_{0}^{2}(\mu, h), \quad n_{s}(\mu, h)=y_{s}^{-2}(\mu, h)$.

In particular, taking $h \rightarrow 0$ with $\mu$ general, we again find the anticipated universal behavior at large $s$,

$$
\lim _{h \rightarrow 0} n_{s}(\mu, h)=\frac{\left(1+e^{\mu}\right)^{2}}{1+s\left(1+e^{\mu}\right)^{2}} \sim 1 / s^{2} .
$$

The dressed electronic charge in the high-temperature limit and general $h$ and $\mu$ is thus

$n_{0}^{\mathrm{dr}}(\mu, h)=-\partial_{\mu} \log \mathcal{Y}_{0}(h, \mu)=\frac{1+e^{2 h}+2 e^{h+\mu}}{1+e^{2 h}+e^{h+\mu}}$,

and, as already emphasized previously, is manifestly strictly positive. On the other hand, the dressed magnetization is

$m_{0}^{\mathrm{dr}}(\mu, h)=-\partial_{h} \log \mathcal{Y}_{0}(h, \mu)=\frac{e^{2 h}-1}{1+e^{2 h}+e^{h+\mu}}$,

which vanishes in the $h \rightarrow 0$ limit, namely, $m^{\operatorname{dr}}(\mu, h)=$ $\left(2 / 2+e^{\mu}\right) h+\mathcal{O}\left(h^{3}\right)$. The dressed magnetization for the $s$ strings displays an analogous qualitative behavior; in the $\mu \rightarrow 0$ limit, we find $\lim _{\mu \rightarrow 0} m_{s}^{\mathrm{dr}}(\mu, h)=$ $\frac{1}{6}(2 s+1)^{2} h+\mathcal{O}\left(h^{3}\right)$.
Next, we consider the dressed dispersion relations for the quasiparticles. The dressing equations can be put in the following compact algebraic form:

$$
\begin{gathered}
F_{0}=d_{0}+\left(K_{1} \star s\right) \star n_{0} F_{0}-s \star \bar{n}_{1} F_{1}, \\
F_{1}=s \star\left(n_{0} F_{0}+\bar{n}_{2} F_{2}\right), \\
F_{s \geq 2}=s \star\left(\bar{n}_{s-1} F_{s-1}+\bar{n}_{s+1} F_{s+1}\right) .
\end{gathered}
$$

Specifically, for $F_{A} \rightarrow \partial_{\theta} \varepsilon_{A}$ with $d_{0} \rightarrow \partial_{\theta} e_{0}(\theta)=2 \theta$, one obtains the dressed energy derivatives, and likewise for $F_{A} \rightarrow \rho_{A}^{\text {tot }}$ with $d_{0}=1 / 2 \pi$, the total state densities. Recall that these are related to the dressed momentum derivatives via $\left[p_{A}^{\prime}(\theta)\right]^{\mathrm{dr}}=2 \pi \rho_{A}^{\text {tot }}(\theta)$. Despite the UV catastrophe, the rapidity densities are well defined in the $\beta \rightarrow 0$ limit where they (in distinction to the NLSM) become flat.

The solution to the homogeneous "bulk" recurrence relation, $\rho_{s}^{\text {tot }}=\frac{1}{2}\left(\bar{n}_{s-1} \rho_{s-1}^{\text {tot }}+\bar{n}_{s+1} \rho_{s+1}^{\text {tot }}\right)$, with the prescribed large- $s$ asymptotics, reads

$$
\rho_{s \geq 1}^{\mathrm{tot}}=\frac{\alpha}{2 \pi}\left(\frac{y_{s}}{y_{s-1}}-\frac{y_{s}}{y_{s+1}}\right) .
$$

This formula is in fact the same bulk solution governing the Heisenberg $\mathrm{SU}(2)$ chain upon replacing $\mathfrak{g} \mathfrak{u}(2)$ characters $\chi_{s}(h)$ with $y_{s}(\mu, h)$. The difference shows up in the initial conditions, i.e., in the equations for densities $\rho_{0}^{\text {tot }}$ and $\rho_{1}^{\text {tot }}$, which provide two extra equations to uniquely determine the unknown function $\rho_{0}^{\text {tot }}$ and coefficient $\alpha$. We find $\alpha=-\left(y_{-1} y_{0}\right) / 2 \pi=a c /[(a+c)(1+a c)]$, and

$$
\rho_{0}^{\mathrm{tot}}=\frac{1}{2 \pi} \frac{\left(a^{2}-1\right)\left(b^{2}+1\right)}{1-a^{2} b^{2}} .
$$

In the $h \rightarrow 0$ limit, the latter simplifies to

$\rho_{s}^{\mathrm{tot}} \rightarrow \frac{2 c(c+s+c s)}{(1+c)[s(c+1)-1][s(c+1)+2 c+1]}$,

whence we can extract the anticipated scaling at large $s$,

$$
\rho_{s}^{\mathrm{tot}}(\mu, h)=\frac{2 e^{\mu}}{\left(1+e^{\mu}\right)^{2}} \frac{1}{s}+\mathcal{O}\left(1 / s^{2}\right) .
$$

Furthermore, for $\mu \rightarrow 0$, the result is even simpler: $\lim _{h \rightarrow 0} \rho_{s}^{\text {tot }}(0, h)=(2 s+1) /\left(4 s^{2}+4 s-3\right)$.

Since the bare energies are not UV-finite quantities, the corresponding dressed energies are well defined only at finite inverse temperature $\beta$. Similarly as in other QFTs, the solutions to the dressing equations cannot be found in a closed algebraic form; instead, one has to resort to numerical solutions. We find the anticipated scaling relation for the effective velocities of giant magnons at large $s$, 


$$
v_{s}^{\mathrm{eff}} \sim 1 / s
$$

irrespectively of the coupling $c>0$. This result can, in fact, be consistently deduced from the strong-coupling limit $c \rightarrow \infty$, where the electron excitations effectively decouple from magnons; the latter interact precisely as spins in the isotropic Heisenberg spin- $1 / 2$ chain [up to $\mathcal{O}(1 / c)$ corrections], which, themselves, already give rise to superdiffusive magnetization transport at any finite density at half filling.

[1] R. Nandkishore and D. A. Huse, Many-Body Localization and Thermalization in Quantum Statistical Mechanics, Annu. Rev. Condens. Matter Phys. 6, 15 (2015).

[2] R. Vasseur and J. E. Moore, Nonequilibrium Quantum Dynamics and Transport: From Integrability to ManyBody Localization, J. Stat. Mech. (2016) 064010.

[3] D. A. Abanin, E. Altman, I. Bloch, and M. Serbyn, Colloquium: Many-Body Localization, Thermalization, and Entanglement, Rev. Mod. Phys. 91, 021001 (2019).

[4] J. M. Deutsch, Quantum Statistical Mechanics in a Closed System, Phys. Rev. A 43, 2046 (1991).

[5] M. Srednicki, Chaos and Quantum Thermalization, Phys. Rev. E 50, 888 (1994).

[6] M. Rigol, V. Dunjko, V. Yurovsky, and M. Olshanii, Relaxation in a Completely Integrable Many-Body Quantum System: An Ab Initio Study of the Dynamics of the Highly Excited States of 1D Lattice Hard-Core Bosons, Phys. Rev. Lett. 98, 050405 (2007).

[7] L. D'Alessio, Y. Kafri, A. Polkovnikov, and M. Rigol, From Quantum Chaos and Eigenstate Thermalization to Statistical Mechanics and Thermodynamics, Adv. Phys. 65, 239 (2016).

[8] L. Vidmar and M. Rigol, Generalized Gibbs Ensemble in Integrable Lattice Models, J. Stat. Mech. (2016) 064007.

[9] E. Ilievski, M. Medenjak, T. Prosen, and L. Zadnik, Quasilocal Charges in Integrable Lattice Systems, J. Stat. Mech. (2016) 064008.

[10] H. Castella, X. Zotos, and P. Prelovšek, Integrability and Ideal Conductance at Finite Temperatures, Phys. Rev. Lett. 74, 972 (1995).

[11] B. Bertini, F. Heidrich-Meisner, C. Karrasch, T. Prosen, R. Steinigeweg, and M. Žnidarič, Finite-Temperature Transport in One-Dimensional Quantum Lattice Models, Rev. Mod. Phys. 93, 025003 (2021).

[12] A. B. Zamolodchikov and A. B. Zamolodchikov, Relativistic Factorized S-Matrix in Two Dimensions Having $O(N)$ Isotopic Symmetry, Nucl. Phys. B133, 525 (1978).

[13] A. B. Zamolodchikov and A. B. Zamolodchikov, Factorized S-Matrices in Two Dimensions as the Exact Solutions of Certain Relativistic Quantum Field Theory Models, Ann. Phys. (N.Y.) 120, 253 (1979).

[14] C. N. Yang and C.P. Yang, Thermodynamics of a One-Dimensional System of Bosons with Repulsive Delta-Function Interaction, J. Math. Phys. (N.Y.) 10, 1115 (1969).
[15] O. A. Castro-Alvaredo, B. Doyon, and T. Yoshimura, Emergent Hydrodynamics in Integrable Quantum Systems Out of Equilibrium, Phys. Rev. X 6, 041065 (2016).

[16] B. Bertini, M. Collura, J. De Nardis, and M. Fagotti, Transport in Out-of-Equilibrium XXZ Chains: Exact Profiles of Charges and Currents, Phys. Rev. Lett. 117, 207201 (2016).

[17] E. Ilievski and J. De Nardis, Microscopic Origin of Ideal Conductivity in Integrable Quantum Models, Phys. Rev. Lett. 119, 020602 (2017).

[18] B. Doyon and H. Spohn, Drude Weight for the LiebLiniger Bose Gas, SciPost Phys. 3, 039 (2017).

[19] E. Ilievski and J. De Nardis, Ballistic Transport in the One-Dimensional Hubbard Model: The Hydrodynamic Approach, Phys. Rev. B 96, 081118(R) (2017).

[20] V. B. Bulchandani, R. Vasseur, C. Karrasch, and J. E. Moore, Bethe-Boltzmann Hydrodynamics and Spin Transport in the XXZ Chain, Phys. Rev. B 97, 045407 (2018).

[21] V. B. Bulchandani, R. Vasseur, C. Karrasch, and J.E. Moore, Solvable Hydrodynamics of Quantum Integrable Systems, Phys. Rev. Lett. 119, 220604 (2017).

[22] B. Doyon, T. Yoshimura, and J.-S. Caux, Soliton Gases and Generalized Hydrodynamics, Phys. Rev. Lett. 120, 045301 (2018).

[23] J.-S. Caux, B. Doyon, J. Dubail, R. Konik, and T. Yoshimura, Hydrodynamics of the Interacting Bose Gas in the Quantum Newton Cradle Setup, SciPost Phys. 6, 070 (2019).

[24] A. Bastianello, J. D. Nardis, and A. D. Luca, Generalized Hydrodynamics with Dephasing Noise, Phys. Rev. B 102, 161110 (2020).

[25] A. Bastianello, V. Alba, and J.-S. Caux, Generalized Hydrodynamics with Space-Time Inhomogeneous Interactions, Phys. Rev. Lett. 123, 130602 (2019).

[26] Notice that even when a quantity exhibits ballistic transport, the subleading corrections to ballistic dynamics (seen, e.g., in the low-frequency conductivity) can be anomalous $[27,28]$.

[27] S. Gopalakrishnan, R. Vasseur, and B. Ware, Anomalous Relaxation and the High-Temperature Structure Factor of XXZ Spin Chains, Proc. Natl. Acad. Sci. U.S.A. 116, 16250 (2019).

[28] U. Agrawal, S. Gopalakrishnan, R. Vasseur, and B. Ware, Anomalous Low-Frequency Conductivity in Easy-Plane XXZ Spin Chains, Phys. Rev. B 101, 224415 (2020).

[29] M. Žnidarič, Spin Transport in a One-Dimensional Anisotropic Heisenberg Model, Phys. Rev. Lett. 106, 220601 (2011).

[30] M. Ljubotina, M. Žnidarič, and T. Prosen, Kardar-ParisiZhang Physics in the Quantum Heisenberg Magnet, Phys. Rev. Lett. 122, 210602 (2019).

[31] M. Kardar, G. Parisi, and Y.-C. Zhang, Dynamic Scaling of Growing Interfaces, Phys. Rev. Lett. 56, 889 (1986).

[32] M. Prähofer and H. Spohn, Exact Scaling Functions for One-Dimensional Stationary KPZ Growth, J. Stat. Phys. 115, 255 (2004).

[33] T. Sasamoto and H. Spohn, One-Dimensional KardarParisi-Zhang Equation: An Exact Solution and Its Universality, Phys. Rev. Lett. 104, 230602 (2010). 
[34] P. Calabrese and P. L. Doussal, Exact Solution for the Kardar-Parisi-Zhang Equation with Flat Initial Conditions, Phys. Rev. Lett. 106, 250603 (2011).

[35] I. Corwin, The Kardar-Parisi-Zhang Equation and Universality Class, Random Matrices Theory Appl. 01, 1130001 (2012).

[36] K. A. Takeuchi, An Appetizer to Modern Developments on the Kardar-Parisi-Zhang Universality Class, Physica (Amsterdam) 504A, 77 (2018).

[37] M. Dupont and J. E. Moore, Universal Spin Dynamics in Infinite-Temperature One-Dimensional Quantum Magnets, Phys. Rev. B 101, 121106 (2020).

[38] M. Fava, B. Ware, S. Gopalakrishnan, R. Vasseur, and S. A. Parameswaran, Spin Crossovers and Superdiffusion in the One-Dimensional Hubbard Model, Phys. Rev. B 102, 115121 (2020).

[39] T. Prosen and B. Žunkovič, Macroscopic Diffusive Transport in a Microscopically Integrable Hamiltonian System, Phys. Rev. Lett. 111, 040602 (2013).

[40] A. Das, M. Kulkarni, H. Spohn, and A. Dhar, KardarParisi-Zhang Scaling for an Integrable Lattice LandauLifshitz Spin Chain, Phys. Rev. E 100, 042116 (2019).

[41] Ž. Krajnik and T. Prosen, Kardar-Parisi-Zhang Physics in Integrable Rotationally Symmetric Dynamics on Discrete Space-Time Lattice, J. Stat. Phys. 179, 110 (2020).

[42] Ž. Krajnik, E. Ilievski, and T. Prosen, Integrable Matrix Models in Discrete Space-Time, SciPost Phys. 9, 038 (2020).

[43] S. Gopalakrishnan and R. Vasseur, Kinetic Theory of Spin Diffusion and Superdiffusion in XXZ Spin Chains, Phys. Rev. Lett. 122, 127202 (2019).

[44] J. De Nardis, S. Gopalakrishnan, E. Ilievski, and R. Vasseur, Superdiffusion from Emergent Classical Solitons in Quantum Spin Chains, Phys. Rev. Lett. 125, 070601 (2020).

[45] V. B. Bulchandani, Kardar-Parisi-Zhang Universality from Soft Gauge Modes, Phys. Rev. B 101, 041411 (2020).

[46] E. Ilievski, J. De Nardis, M. Medenjak, and T. Prosen, Superdiffusion in One-Dimensional Quantum Lattice Models, Phys. Rev. Lett. 121, 230602 (2018).

[47] C. K. Lai, Lattice Gas with Nearest-Neighbor Interaction in One Dimension with Arbitrary Statistics, J. Math. Phys. (N.Y.) 15, 1675 (1974).

[48] B. Sutherland, Model for a Multicomponent Quantum System, Phys. Rev. B 12, 3795 (1975).

[49] M. Kulkarni, D. A. Huse, and H. Spohn, Fluctuating Hydrodynamics for a Discrete Gross-Pitaevskii Equation: Mapping onto the Kardar-Parisi-Zhang Universality Class, Phys. Rev. A 92, 043612 (2015).

[50] A. Das, K. Damle, A. Dhar, D. A. Huse, M. Kulkarni, C. B. Mendl, and H. Spohn, Nonlinear Fluctuating Hydrodynamics for the Classical XXZ Spin Chain, J. Stat. Phys. 180, 238 (2020).

[51] V. Popkov, A. Schadschneider, J. Schmidt, and G. M. Schtz, Fibonacci Family of Dynamical Universality Classes, Proc. Natl. Acad. Sci. U.S.A. 112, 12645 (2015).

[52] M. Žnidarič, Magnetization Transport in Spin Ladders and Next-Nearest-Neighbor Chains, Phys. Rev. B 88, 205135 (2013).

[53] A. V. Gorshkov, M. Hermele, V. Gurarie, C. Xu, P. S. Julienne, J. Ye, P. Zoller, E. Demler, M. D. Lukin, and
A. M. Rey, Two-Orbital $S U(N)$ Magnetism with Ultracold Alkaline-Earth Atoms, Nat. Phys. 6, 289 (2010).

[54] M. Hermele, V. Gurarie, and A. M. Rey, Mott Insulators of Ultracold Fermionic Alkaline Earth Atoms: Underconstrained Magnetism and Chiral Spin Liquid, Phys. Rev. Lett. 103, 135301 (2009).

[55] E. Dagotto and T. M. Rice, Surprises on the Way from One- to Two-Dimensional Quantum Magnets: The Ladder Materials, Science 271, 618 (1996).

[56] M. T. Batchelor, X.-W. Guan, A. Foerster, and H.-Q. Zhou, Note on the Thermodynamic Bethe Ansatz Approach to the Quantum Phase Diagram of the Strong Coupling Ladder Compounds, New J. Phys. 5, 107 (2003).

[57] M. T. Batchelor, X. W. Guan, N. Oelkers, and Z. Tsuboi, Integrable Models and Quantum Spin Ladders: Comparison between Theory and Experiment for the Strong Coupling Ladder Compounds, Adv. Phys. 56, 465 (2007).

[58] See Supplemental Material at http://link.aps.org/ supplemental/10.1103/PhysRevX.11.031023 for the technical background on the algebraic Bethe Ansatz and other group-theoretical concepts.

[59] A. Zamolodchikov and A. Zamolodchikov, Massless Factorized Scattering and Sigma Models with Topological Terms, Nucl. Phys. B379, 602 (1992).

[60] A. N. Kirillov and N. Y. Reshetikhin, The Yangians, Bethe Ansatz and Combinatorics, Lett. Math. Phys. 12, 199 (1986).

[61] A. N. Kirillov, Identities for the Rogers Dilogarithm Function Connected with Simple Lie Algebras, J. Sov. Math. 47, 2450 (1989).

[62] A. N. Kirillov and N. Y. Reshetikhin, Representations of Yangians and Multiplicities of Occurrence of the Irreducible Components of the Tensor Product of Representations of Simple Lie Algebras, J. Sov. Math. 52, 3156 (1990).

[63] A. Kuniba, T. Nakanishi, and J. Suzuki, T-Systems and Y-Systems in Integrable Systems, J. Phys. A 44, 103001 (2011).

[64] A. Kuniba and T. Nakanishi, Spectra in Conformal Field Theories From the Rogers Dilogarithm, Mod. Phys. Lett. A 07, 3487 (1992).

[65] A. Kuniba, T. Nakaishi, and J. Suzuki, Characters in Conformal Field Theories from Thermodynamic Bethe Ansatz, Mod. Phys. Lett. A 08, 1649 (1993).

[66] A. Kuniba, T. Nakanishi, and J. Suzuki, Functional Relations in Solvable Lattice Models I: Functional Relations and Representation Theory, Int. J. Mod. Phys. A 09, 5215 (1994).

[67] L. Faddeev, N. Reshetikhin, and L. Takhtajan, Quantization of Lie Groups and Lie Algebras, in Algebraic Analysis (Elsevier, New York, 1988), pp. 129-139.

[68] V. G. Drinfel'd, Quantum Groups, J. Sov. Math. 41, 898 (1988).

[69] V. G. Drinfed, Hopf Algebras and the Quantum YangBaxter Equation, in Yang-Baxter Equation in Integrable Systems (World Scientific, Singapore, 1990), pp. 264-268.

[70] I. Krichever, O. Lipan, P. Wiegmann, and A. Zabrodin, Quantum Integrable Models and Discrete Classical Hirota Equations, Commun. Math. Phys. 188, 267 (1997). 
[71] A. V. Zabrodin, Hirota Equation and Bethe Ansatz, Theor. Math. Phys. 116, 782 (1998).

[72] M. Takahashi, One-Dimensional Hubbard Model at Finite Temperature, Prog. Theor. Phys. 47, 69 (1972).

[73] F. H. L. Essler, H. Frahm, F. Göhmann, A. Klümper, and V.E. Korepin, The One-Dimensional Hubbard Model (Cambridge University Press, Cambridge, England, 2005).

[74] S. Belliard and E. Ragoucy, The Nested Bethe Ansatz for 'All' Closed Spin Chains, J. Phys. A 41, 295202 (2008).

[75] N. Gromov, F. Levkovich-Maslyuk, and G. Sizov, New Construction of Eigenstates and Separation of Variables for $S U(N)$ Quantum Spin Chains, J. High Energy Phys. 09 (2017) 111.

[76] F. Levkovich-Maslyuk, The Bethe Ansatz, J. Phys. A 49, 323004 (2016).

[77] N. A. Slavnov, Oscillator Construction of $s u(n \mid m) Q$ Operators, SciPost Phys. Lect. Notes 19 (2020).

[78] V. V. Bazhanov, T. Łukowski, C. Meneghelli, and M. Staudacher, A Shortcut to the Q-Operator, J. Stat. Mech. (2010) P11002.

[79] R. Frassek, T. Łukowski, C. Meneghelli, and M. Staudacher, Oscillator Construction of Q-Operators, Nucl. Phys. B850, 175 (2011).

[80] R. Frassek, Oscillator Realisations Associated to the D-Type Yangian: Towards the Operatorial Q-System of Orthogonal Spin Chains, Nucl. Phys. B956, 115063 (2020).

[81] E. K. Sklyanin, Separation of Variables, Prog. Theor. Phys. Suppl. 118, 35 (1995).

[82] M. Jimbo and T. Miwa, Solitons and Infinite-Dimensional Lie Algebras, Publ. RIMS 19, 943 (1983).

[83] R. Hirota, Nonlinear Partial Difference Equations. II. Discrete-Time Toda Equation, J. Phys. Soc. Jpn. 43, 2074 (1977).

[84] R. Hirota, The Direct Method in Soliton Theory, edited by A. Nagai, J. Nimmo, and C. Gilson (Cambridge University Press, Cambridge, England, 2004).

[85] L. Faddeev and A. Y. Volkov, Hirota Equation as an Example of an Integrable Symplectic Map, Lett. Math. Phys. 32, 125 (1994).

[86] V. Kazakov, S. Leurent, and D. Volin, T-System on THook: Grassmannian Solution and Twisted Quantum Spectral Curve, J. High Energy Phys. 12 (2016) 044.

[87] D. Volin, String Hypothesis for Spin Chains: A Particlel Hole Democracy, Lett. Math. Phys. 102, 1 (2012).

[88] A. Vladimirov, Non-String Two-Magnon Configurations in the Isotropic Heisenberg Magnet, Phys. Lett. A 105, 418 (1984).

[89] K. Isler and M. Paranjape, Violations of the String Hypothesis in the Solutions of the Bethe Ansatz Equations in the XXX-Heisenberg Model, Phys. Lett. B 319, 209 (1993).

[90] D. Hernandez, Kirillov-Reshetikhin Conjecture: The General Case, Int. Math. Res. Not. 10.1093/imrn/rnp121 (2009).

[91] D. Chernyak, S. Leurent, and D. Volin, Completeness of Wronskian Bethe Equations for Rational gl(m|n) Spin Chain, arXiv:2004.02865.

[92] A. Klümper and P. A. Pearce, Conformal Weights of RSOS Lattice Models and Their Fusion Hierarchies, Physica (Amsterdam) 183A, 304 (1992).
[93] G. Hatayama, A. Kuniba, M. Okado, T. Takagi, and Y. Yamada, Remarks on Fermionic Formula (1999).

[94] H. Nakajima, t-Analogs of q-Characters of KirillovReshetikhin Modules of Quantum Affine Algebras, Represent. Theory Am. Math. Soc. 7, 259 (2003).

[95] M. Takahashi, Thermodynamics of the Heisenberg-Ising Model for $|\Delta|<1$ in One Dimension, Phys. Lett. A 36, 325 (1971).

[96] M. Gaudin, Thermodynamics of the Heisenberg-Ising Ring for $\Delta>1$, Phys. Rev. Lett. 26, 1301 (1971).

[97] M. Mestyán and B. Pozsgay, Short Distance Correlators in the XXZ Spin Chain for Arbitrary String Distributions, J. Stat. Mech. (2014) P09020.

[98] M. Mestyán, B. Pozsgay, G. Takács, and M. A. Werner, Quenching the XXZ Spin Chain: Quench Action Approach Versus Generalized Gibbs Ensemble, J. Stat. Mech. (2015) P04001.

[99] B. Pozsgay, E. Vernier, and M. A. Werner, On Generalized Gibbs Ensembles with an Infinite Set of Conserved Charges, J. Stat. Mech. (2017) 093103.

[100] E. Ilievski, J. De Nardis, B. Wouters, J.-S. Caux, F. H. L. Essler, and T. Prosen, Complete Generalized Gibbs Ensembles in an Interacting Theory, Phys. Rev. Lett. 115, 157201 (2015).

[101] E. Ilievski, E. Quinn, J. D. Nardis, and M. Brockmann, String-Charge Duality in Integrable Lattice Models, J. Stat. Mech. (2016) 063101.

[102] G. Fehér and B. Pozsgay, Generalized Gibbs Ensemble and String-Charge Relations in Nested Bethe Ansatz, SciPost Phys. 8, 034 (2020).

[103] B. Wouters, J. D. Nardis, M. Brockmann, D. Fioretto, M. Rigol, and J.-S. Caux, Quenching the Anisotropic Heisenberg Chain: Exact Solution and Generalized Gibbs Ensemble Predictions, Phys. Rev. Lett. 113, 117202 (2014).

[104] E. Ilievski, M. Medenjak, and T. Prosen, Quasilocal Conserved Operators in the Isotropic Heisenberg Spin1/2 Chain, Phys. Rev. Lett. 115, 120601 (2015).

[105] E. Ilievski, E. Quinn, and J.-S. Caux, From Interacting Particles to Equilibrium Statistical Ensembles, Phys. Rev. B 95, 115128 (2017).

[106] E. Ilievski and E. Quinn, The Equilibrium Landscape of the Heisenberg Spin Chain, SciPost Phys. 7, 033 (2019).

[107] V. E. Korepin, N. M. Bogoliubov, and A. G. Izergin, Quantum Inverse Scattering Method and Correlation Functions, Cambridge Monographs on Mathematical Physics (Cambridge University Press, Cambridge, England, 1993).

[108] E. Quinn and S. Frolov, Excited States in Bethe Ansatz. Solvable Models and the Dressing of Spin and Charge, J. Phys. A 46, 205001 (2013).

[109] J. De Nardis, M. Medenjak, C. Karrasch, and E. Ilievski, Anomalous Spin Diffusion in One-Dimensional Antiferromagnets, Phys. Rev. Lett. 123, 186601 (2019).

[110] J. De Nardis, D. Bernard, and B. Doyon, Hydrodynamic Diffusion in Integrable Systems, Phys. Rev. Lett. 121, 160603 (2018).

[111] S. Gopalakrishnan, D. A. Huse, V. Khemani, and R. Vasseur, Hydrodynamics of Operator Spreading and Quasiparticle Diffusion in Interacting Integrable Systems, Phys. Rev. B 98, 220303 (2018). 
[112] J. De Nardis, D. Bernard, and B. Doyon, Diffusion in Generalized Hydrodynamics and Quasiparticle Scattering, SciPost Phys. 6, 049 (2019).

[113] Ž. Krajnik, E. Ilievski, and T. Prosen, Undular Diffusion in Nonlinear Sigma Models, Phys. Rev. Lett. 125, 240607 (2020).

[114] B. Doyon, Lecture notes on Generalised Hydrodynamics, SciPost Phys. Lect. Notes 18 (2020).

[115] M. Borsi, B. Pozsgay, and L. Pristyák, Current Operators in Bethe Ansatz and Generalized Hydrodynamics: An Exact Quantum-Classical Correspondence, Phys. Rev. X 10, 011054 (2020).

[116] B. Pozsgay, Algebraic Construction of Current Operators in Integrable Spin Chains, Phys. Rev. Lett. 125, 070602 (2020).

[117] X. Zotos, Finite Temperature Drude Weight of the OneDimensional Spin-1/2 Heisenberg Model, Phys. Rev. Lett. 82, 1764 (1999).

[118] E. Ilievski and T. Prosen, Thermodyamic Bounds on Drude Weights in Terms of Almost-Conserved Quantities, Commun. Math. Phys. 318, 809 (2013).

[119] Note that the factor of 2 in Eq. (20) follows from the familiar scaling of the variance $\delta x^{2}=2 D t$ of a diffusing particle in one dimension.

[120] J. D. Nardis, M. Medenjak, C. Karrasch, and E. Ilievski, Universality Classes of Spin Transport in OneDimensional Isotropic Magnets: The Onset of Logarithmic Anomalies, Phys. Rev. Lett. 124, 210605 (2020).

[121] D. Bernard, An Introduction to Yangian Symmetries, Int. J. Mod. Phys. B 07, 3517 (1993).

[122] N. J. MacKay, Introduction to Yangian Symmetry in Integrable Field Theory, Int. J. Mod. Phys. A 20, 7189 (2005).

[123] G. Vidal, Efficient Classical Simulation of Slightly Entangled Quantum Computations, Phys. Rev. Lett. 91, 147902 (2003).

[124] F. Verstraete, J. J. García-Ripoll, and J. I. Cirac, Matrix Product Density Operators: Simulation of FiniteTemperature and Dissipative Systems, Phys. Rev. Lett. 93, 207204 (2004).

[125] C. Karrasch, J. H. Bardarson, and J.E. Moore, FiniteTemperature Dynamical Density Matrix Renormalization Group and the Drude Weight of Spin-1/2 Chains, Phys. Rev. Lett. 108, 227206 (2012).

[126] L. Takhtajan, Integration of the Continuous Heisenberg Spin Chain through the Inverse Scattering Method, Phys. Lett. A 64, 235 (1977).

[127] M. Lakshmanan, T. Ruijgrok, and C. Thompson, On the Dynamics of a Continuum Spin System, Physica (Amsterdam) 84A, 577 (1976).

[128] H. Watanabe and H. Murayama, Unified Description of Nambu-Goldstone Bosons without Lorentz Invariance, Phys. Rev. Lett. 108, 251602 (2012).

[129] H. Watanabe and H. Murayama, Redundancies in NambuGoldstone Bosons, Phys. Rev. Lett. 110, 181601 (2013).

[130] Y. Hidaka, Counting Rule for Nambu-Goldstone Modes in Nonrelativistic Systems, Phys. Rev. Lett. 110, 091601 (2013).

[131] H. Nielsen and S. Chadha, On How to Count Goldstone Bosons, Nucl. Phys. B105, 445 (1976).
[132] B. Stefanski and A. Tseytlin, Large Spin Limits of AdS/ CFT and Generalized Landau-Lifshitz Equations, J. High Energy Phys. 05 (2004) 042.

[133] B. Stefański, Landau-Lifshitz Sigma-Models, Fermions and the AdS/CFT Correspondence, J. High Energy Phys. 07 (2007) 009.

[134] A. Perelomov, Coherent States for Arbitrary Lie Groups, in Generalized Coherent States and Their Applications (Springer, Berlin, Heidelberg, 1986), pp. 40-47.

[135] A. Dhar and B. S. Shastry, Bloch Walls and Macroscopic String States in Bethe's Solution of the Heisenberg Ferromagnetic Linear Chain, Phys. Rev. Lett. 85, 2813 (2000).

[136] V. A. Kazakov, A. Marshakov, J. A. Minahan, and K. Zarembo, Classical/Quantum Integrability in AdS/CFT, J. High Energy Phys. 05 (2004) 024.

[137] T. Bargheer, N. Beisert, and N. Gromov, Quantum Stability for the Heisenberg Ferromagnet, New J. Phys. 10, 103023 (2008).

[138] S. Schäfer-Nameki, Review of AdS/CFT Integrability, Chapter II.4: The Spectral Curve, Lett. Math. Phys. 99, 169 (2012).

[139] B. A. Dubrovin, I. M. Krichever, and S. P. Novikov, Integrable Systems. I, in Dynamical Systems IV (Springer, Berlin, Heidelberg, 2001), pp. 177-332.

[140] N. Beisert, V. Kazakov, and K. Sakai, Algebraic Curve for the $S O(6)$ Sector of AdS/CFT, Commun. Math. Phys. 263, 611 (2006).

[141] N. Beisert, V. Kazakov, K. Sakai, and K. Zarembo, The Bethe-Ansatz for $\mathcal{N}=4$ Super Yang-Mills, J. Commun. Math. Phys. 263, 659 (2006).

[142] N. Gromov and P. Vieira, Complete 1-Loop Test of AdS/ CFT, J. High Energy Phys. 04 (2008) 046.

[143] J. A. Minahan and K. Zarembo, The Bethe-Ansatz for Script $N=4$ Super Yang-Mills, J. High Energy Phys. 03 (2003) 013.

[144] N. Beisert, C. Ahn, L. F. Alday, Z. Bajnok, J. M. Drummond, L. Freyhult, N. Gromov, R. A. Janik, V. Kazakov, T. Klose et al., Review of AdS/CFT Integrability: An Overview, Lett. Math. Phys. 99, 3 (2012).

[145] B. Vicedo, Giant Magnons and Singular Curves, J. High Energy Phys. 12 (2007) 078.

[146] V. Kazakov, A. Sorin, and A. Zabrodin, Supersymmetric Bethe Ansatz and Baxter Equations from Discrete Hirota Dynamics, Nucl. Phys. B790, 345 (2008).

[147] E. Ilievski, Dissipation-driven integrable fermionic systems: from graded Yangians to exact nonequilibrium steady states, SciPost Phys. 3, 031 (2017).

[148] A. Kuniba, Quantum R Matrix for $\mathrm{G}_{2}$ and a Solvable 175Vertex Model, J. Phys. A 23, 1349 (1990).

[149] P. Fendley and H. Saleur, Nonequilibrium dc Noise in a Luttinger Liquid with an Impurity, Phys. Rev. B 54, 10845 (1996).

[150] A. Polyakov and P. Wiegmann, Theory of Nonabelian Goldstone Bosons in Two Dimensions, Phys. Lett. B 131, 121 (1983).

[151] P. Wiegmann, On the Theory of Nonabelian Goldstone Bosons in Two Dimensions; Exact Solution of the $S U(N) \otimes S U(N)$ Nonlinear $\sigma$ Model, Phys. Lett. B 141, 217 (1984). 
[152] P. Wiegmann, Exact Factorized S-Matrix of the Chiral Field in Two Dimensions, Phys. Lett. B 142, 173 (1984).

[153] L. Faddeev and N. Reshetikhin, Integrability of the Principal Chiral Field Model in $1+1$ Dimension, Ann. Phys. (N.Y.) 167, 227 (1986).

[154] E. Ogievetsky, N. Reshetikhin, and P. Wiegmann, The Principal Chiral Field in Two Dimensions on Classical Lie Algebras: The Bethe-Ansatz. Solution and Factorized Theory of Scattering, Nucl. Phys. B280, 45 (1987).

[155] V. Kazakov and S. Leurent, Finite Size Spectrum of $S U(N)$ Principal Chiral Field from Discrete Hirota Dynamics, Nucl. Phys. B902, 354 (2016).

[156] P. Fendley, Integrable Sigma Models and Perturbed Coset Models, J. High Energy Phys. 05 (2001) 050.

[157] C. N. Yang, Some Exact Results for the Many-Body Problem in One Dimension with Repulsive Delta-Function Interaction, Phys. Rev. Lett. 19, 1312 (1967).

[158] M. Gaudin, An Interacting System of Fermions in 1d, Phys. Lett. A 24, 55 (1967).

[159] B. Sutherland, Further Results for the Many-Body Problem in One Dimension, Phys. Rev. Lett. 20, 98 (1968).

[160] M. Takahashi, One-Dimensional Electron Gas with DeltaFunction Interaction at Finite Temperature, Prog. Theor. Phys. 46, 1388 (1971).

[161] O. I. Pâțu and A. Klümper, Thermodynamics, Contact, and Density Profiles of the Repulsive Gaudin-Yang Model, Phys. Rev. A 93, 033616 (2016).

[162] M. Mestyán, B. Bertini, L. Piroli, and P. Calabrese, SpinCharge Separation Effects in the Low-Temperature Transport of One-Dimensional Fermi Gases, Phys. Rev. B 99, 014305 (2019).

[163] S. Wang, X. Yin, Y.-Y. Chen, Y. Zhang, and X.-W. Guan, Emergent Ballistic Transport of Bose-Fermi Mixtures in One Dimension, J. Phys. A 53, 464002 (2020).

[164] P. Fendley, Integrable Sigma Models, in Integrable Quantum Field Theories and Their Applications (World Scientific, Singapore, 2001).

[165] S. Fujimoto, Spin Transport Properties of the Quantum One-Dimensional Non-Linear Sigma Model:
An Application to Haldane Gap Systems, J. Phys. Soc. Jpn. 68, 2810 (1999).

[166] S. Sachdev and K. Damle, Low Temperature Spin Diffusion in the One-Dimensional Quantum O(3) Nonlinear $\sigma$ Model, Phys. Rev. Lett. 78, 943 (1997).

[167] S. Sachdev, Universal Relaxational Dynamics Near TwoDimensional Quantum Critical Points, Phys. Rev. B 59, 14054 (1999).

[168] S. Sachdev, Quantum Phase Transitions (Cambridge University Press, Cambridge, England, 2009).

[169] P. Fendley, Integrable Sigma Models with $\theta=\pi$, Phys. Rev. B 63, 104429 (2001).

[170] F. Haldane, Continuum Dynamics of the 1-D Heisenberg Antiferromagnet: Identification with the $O(3)$ Nonlinear Sigma Model, Phys. Lett. A 93, 464 (1983).

[171] A. V. Mikhailov, Relativistically Invariant Models of the Field Theory Integrable by the Inverse Scattering Method, in Twistor Geometry and Non-Linear Systems (Springer, Berlin, Heidelberg, 1982), pp. 186-196.

[172] L. D. Faddeev and L. A. Takhtajan, Hamiltonian Methods in the Theory of Solitons (Springer, Berlin, Heidelberg, 1987).

[173] N. Gromov, V. Kazakov, K. Sakai, and P. Vieira, Strings as Multi-particle States of Quantum Sigma-Models, Nucl. Phys. B764, 15 (2007).

[174] J. Balog and Á. Hegedús, Virial Expansion and TBA in $O(N)$ Sigma-Models, Phys. Lett. B 523, 211 (2001).

[175] N. Gromov, V. Kazakov, and P. Vieira, Finite Volume Spectrum of 2D Field Theories from Hirota Dynamics, J. High Energy Phys. 12 (2009) 060.

[176] V. E. Zakharov and A. V. Mikhailov, On the Integrability of Classical Spinor Models in Two-Dimensional Space-Time, Commun. Math. Phys. 74, 21 (1980).

[177] S. J. van Tongeren, Introduction to the Thermodynamic Bethe Ansatz, J. Phys. A 49, 323005 (2016).

[178] J. H. Perk and C. L. Schultz, New Families of Commuting Transfer Matrices in q-State Vertex Models, Phys. Lett. A 84, 407 (1981).

[179] H. J. de Vega and E. Lopes, Exact Solution of the PerkSchultz Model, Phys. Rev. Lett. 67, 489 (1991). 